\title{
Final Report for Lith 112 High-NA Optics for the Micro-Exposure Tool (MET)
}

J. S. Taylor, P. Gabella, R. Hudyma, G. Sommargren, D. Phillion, M. A. Johnson, R. Soufli, E. Spiller, L. Hale, T. Decker, E. Gullikson, P. Kürz, U.Dinger, G. Seitz, F. Eisert, S. Schulte

\section{December 7, 2001}

U.S. Department of Energy

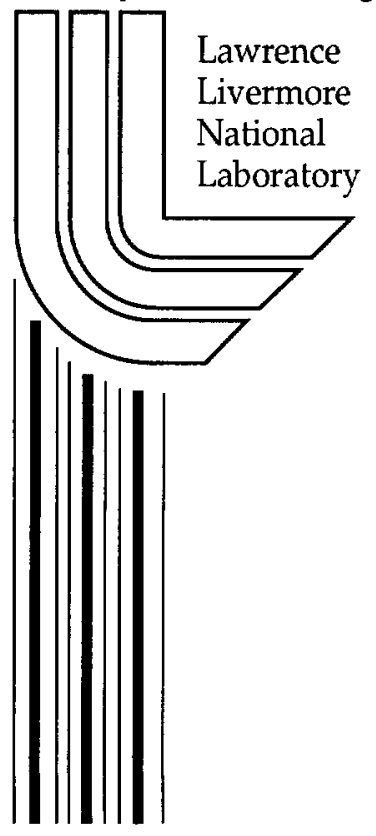




\section{DISCLAIMER}

This document was prepared as an account of work sponsored by an agency of the United States Government. Neither the United States Government nor the University of California nor any of their employees, makes any warranty, express or implied, or assumes any legal liability or responsibility for the accuracy, completeness, or usefulness of any information, apparatus, product, or process disclosed, or represents that its use would not infringe privately owned rights. Reference herein to any specific commercial product, process, or service by trade name, trademark, manufacturer, or otherwise, does not necessarily constitute or imply its endorsement, recommendation, or favoring by the United States Government or the University of California. The views and opinions of authors expressed herein do not necessarily state or reflect those of the United States Government or the University of California, and shall not be used for advertising or product endorsement purposes.

This work was performed under the auspices of the U. S. Department of Energy by the University of California, Lawrence Livermore National Laboratory under Contract No. W-7405-Eng-48.

This report has been reproduced directly from the best available copy.

Available electronically at http://www.doc.gov/bridge

Available for a processing fee to U.S. Department of Energy

And its contractors in paper from

U.S. Department of Energy

Office of Scientific and Technical Information

P.O. Box 62

Oak Ridge, TN 37831-0062

Telephone: (865) 576-8401

Facsimile: (865) 576-5728

E-mail: reports@adonis.osti.gov

Available for the sale to the public from

U.S. Department of Commerce

National Technical Information Service

5285 Port Royal Road

Springfield, VA 22161

Telephone: (800) 553-6847

Facsimile: (703) 605-6900

E-mail: orders@ntis.fedworld.gov

Online ordering: http://www.ntis.gov/ordering.htm

OR

Lawrence Livermore National Laboratory

Technical Information Department's Digital Library

http://www.llnl.gov/tid/Library.html 


\title{
Final Report for Lith 112 \\ High-NA Optics for the Micro-Exposure Tool (MET)
}

\author{
John S. Taylor (jstaylor@llnl.gov) \\ EUV Optics Group Leader \\ Lawrence Livermore National Laboratory
}

\section{ISMT Project Manager:}

\author{
Technical Contributors: \\ Optical Design: \\ Interferometry:
}

Multilayer Coatings:

PO Box Design, Construction:

ALS Scattering, PSD Analysis:

\section{Patricia Gabella (pat.gabella@sematech.org)}

\author{
Russell Hudyma, Paragon Optics (rhudyma@home.com) \\ Gary Sommargren, LLNL (sommargren1@llnl.gov) \\ Donald Phillion, LLNL (phillion1@llnl.gov) \\ Michael A. Johnson, LLNL (mike-johnson@llnl.gov) \\ Regina Soufli, LLNL (souflil@llnl.gov) \\ Eberhard Spiller, LLNL (spiller1@1lnl.gov) \\ Layton Hale, LLNL (hale6@llnl.gov) \\ Todd Decker, LLNL (decker4@1lnl.gov) \\ Eric Gullikson, LBNL (EMGullikson@lbl.gov)
}

Carl Zeiss:

Project Management:

Optics Manufacturing:

Interferometry:

Surface Finish, PSD Analysis:

Alignment:

\author{
Peter Kürz (kuerz@zeiss.de) \\ Udo Dinger \\ Günther Seitz \\ Frank Eisert \\ Stefan Schulte
}

\section{Overview and Background}

Project Lith 112 "High-NA Optics" was initiated in Q3-1999 to lay the groundwork for constructing a small-field imaging system for use in a high-NA micro-exposure tool (MET) to support the development of EUVL resists and for assessing defect printability. ${ }^{1}$ A paradigm had been established at ISMT for resist development for 193-nm and 157-nm lithography, which involved employing micro-exposure tools several years prior to production to serve as platforms for developing resists. Key goals for the MET are to demonstrate the extensibility of EUVL to the 35-nm lithographic node, and to support resist development over multiple EUV generations. Since the fabrication of the optics was viewed as a long-lead activity, proceeding with fabricating the optics was a means of meeting the long-term need for an EUV MET, prior to committing to an exposure platform.

In mid-1998, a contract was placed with Carl Zeiss (Oberkochen, Germany) for fabrication of two aspheric mirror substrates from a VNL optical design. It was considered an important and timely goal to engage Carl Zeiss in EUV optics activities because of the 
clear need for expanded industrial capacity for EUVL substrates. The specifications for figure and finish were a factor of several beyond the current state-of-the-art and initiating MET optics fabrication served as an early engagement by industry in meeting these challenging goals.

The two-mirror EUV imaging system was designed at the VNL and has a numerical aperture (NA) of 0.3 and a $5 x$ reduction ${ }^{2}$. A diagram of the imaging system and key parameters are given in Fig. 1. This design employs two aspheric mirrors that provide a printed field of about $200 \mu \mathrm{m} \times 600 \mu \mathrm{m}$. Because of the need to use a reflective mask with EUVL, the mask is tilted about 5 degrees to the optical axis to enable light to reflect into the system; there is a commensurate tilting of the wafer plane to obtain well-corrected imaging. Extensive modeling has been performed to determine the lithographic performance of this design and demonstrate that the $10 \%$ obscuration will not impact performance. $^{3}$

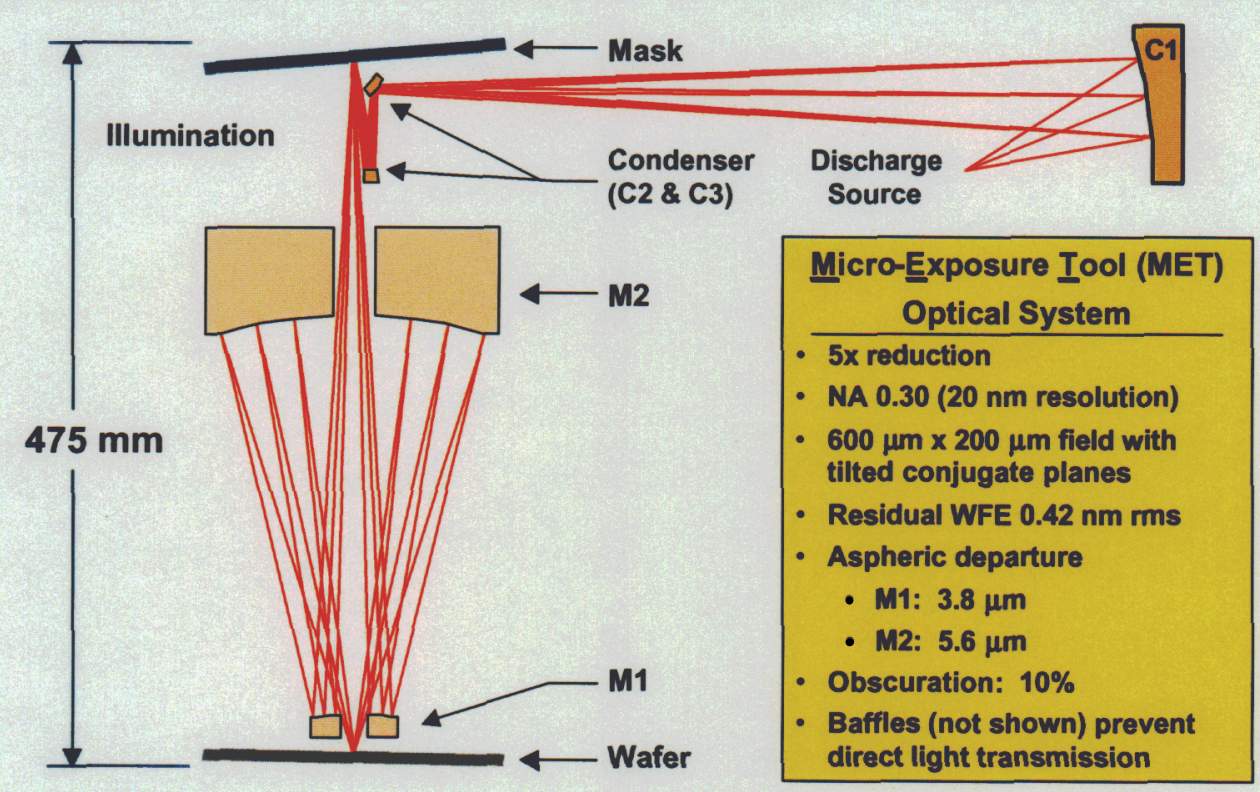

Figure 1. Diagram of MET optical system, shown with potential illuminator geometry.

Lith 112 has spanned about 2 years and encompasses the range of tasks spanning documentation of the optical design through the delivery of an aligned projection optics box (PO Box). A list of the key tasks is given in Table 1. The main goal of this report is to describe progress on Lith 112 during the first three quarters of 2001. The project began with the goal of fabricating a set of multilayer-coated optics, but was extended for a second year to include the design and construction of the PO Box. The fabrication of the optical substrates has proven more challenging than first anticipated, resulting in an overall delay in completing the PO Box. The substrates are expected to be completed and then delivered to the VNL for inspection and coating in Q4-2001. 
Table 1. Tasks for Lith 112 and status.

\begin{tabular}{|l|l|}
\hline Task & Status \\
\hline Document optical design & Done \\
\hline Calculate aerial images & Done \\
\hline Model lithographic performance & Done \\
\hline Formulate substrate specifications & Done \\
\hline Fabricate optical substrates & Set 1 done; Set 2 in-process \\
\hline Formulate multilayer specifications & Done \\
\hline Validate vendor's metrology & Done \\
\hline Deposit multilayer coatings & Set 1 done; waiting for Set 2 \\
\hline Assess quality of ML coatings & Set 1 done; waiting for Set 2 \\
\hline Design PO Box and prepare drawings & Done \\
\hline Fabricate PO Box & Done \\
\hline Fabricate shipping container & Done \\
\hline Assemble coated optics into PO Box & Set 1 done; waiting for Set 2 \\
\hline Mechanically-align PO Box & Set 1 done; waiting for Set 2 \\
\hline Ship assembly to vendor & Set 1 done; waiting for Set 2 \\
\hline Perform interferometric alignment & Set 1 in process \\
\hline Ship aligned PO Box to ISMT or VNL & Waiting for Set 2 \\
\hline $\begin{array}{l}\text { Calculate lithographic performance and } \\
\text { wavefront errors }\end{array}$ & $\begin{array}{l}\text { Waiting for Set } 2 \text { alignment } \\
\text { data }\end{array}$ \\
\hline
\end{tabular}




\section{Status of Optical Fabrication}

The contract with Carl Zeiss comprises the fabrication of aspheric substrates M1 and M2, the metrology of these substrates, and the interferometric alignment of the assembled PO Box. Zeiss also participates with the VNL in the cross-validation of their figure and finish metrology and supplies surrogate spherical substrates for coating development. Our agreement for acquiring the substrates from Zeiss is a collaborative effort where both organizations are maximizing the learning potential of the effort. Zeiss is fabricating two sets of optics, one of which is theirs, and one for International SEMATECH (ISMT). The VNL is constructing two PO Boxes and where one is intended for Zeiss and one for ISMT. In this manner, Zeiss retains an assembled PO Box for their internal EUV development work. The original specifications for the Zeiss and ISMT substrates were nominally identical, but have since evolved.

The date planned for the completion of the two substrates has slipped because of unanticipated difficulties in meeting the challenging figure and finish specifications (described below). However, we have the opportunity to demonstrate key operations in completing a PO Box by immediately employing a set of optics with a relaxed set of specifications for the Zeiss PO Box. For example, we will exercise the plans and equipment for coating the optics with multilayers, assembling the PO Box, shipping the PO Box, and interferometrically aligning the PO Box These initial optics are labeled as "Set 1". The set still under construction and planned for ISMT is referred to as "Set 2". The PO Boxes for housing these optics are referred to as "PO Box 1" and "PO Box 2", respectively.

The specifications for the Set 1 and Set 2 substrates are listed in Table 2, along with the measured values for Set 1 . The mechanical drawings for the MET substrates are documented and have been delivered to ISMT. ${ }^{4,5,6,7}$ The Set 2 specifications are the original requirements established for delivery to ISMT and are the same as for "Optics Set 2" for the Engineering Test Stand. ${ }^{8}$ The specifications comprise three categories: figure, Mid-Spatial-Frequency Roughness (MSFR), and High-Spatial-Frequency Roughness (HSFR). The spatial frequency boundaries for each category are defined in Table 2. The categories of errors are divided approximately according to the types of instruments that would be used to measure them. Figure is measured using full-aperture interferometry, MSFR is measured using phase-shifting interferometric microscopy, and $H S F R$ is measured using an AFM (atomic force microscope). Similarly, the functional requirements that drive these specifications are approximately aligned with these definitions. Figure is associated with wavefront error and resolution, MSFR is associated with flare, and $H S F R$ is associated with throughput loss due to wide-angle scattering outside of the field of view. As will be discussed later, the spatial frequency range that contributes to flare also encompasses a portion of the figure designation.

Various characteristics of the two MET substrates, M1 and M2, are given in Table 3. These substrates are made of Zerodur ${ }^{\circledR}$ to minimize thermal distortion during their manufacturing and in their use in the MET. The primary, M1, is convex with a $54 \mathrm{~mm}$ diameter clear aperture (CA) and M2 is concave with about a $183 \mathrm{~mm} \mathrm{CA}$. There is a hole 
in the center of each optic for beam clearance that has an edge directly abutting the CA. There is a large border outside the CA that extends to the outer diameter, which helps to minimize mounting-induced distortion and offers a freeboard for material removal operations. The amount of aspheric departure is sufficiently large to clearly require advanced aspheric fabrication technology. More significantly, however, the aspheric slope, which defines the rate of change of the aspheric contour with respect to a best-fit sphere, is relatively large and likely to cause added difficulty in manufacturing. In comparison with the substrates for the ETS, the MET M1 has a larger aspheric slope than any of the ETS substrates. Note that one area where the MET substrates may have a simplifying characteristic is that they are circularly symmetric about an axis. The aspheric substrates for the ETS are off-axis elements and the respective pieces of glass are not symmetric about their center.

Zeiss proposed the delivery of an initial set of optics, "Set 1", to a relaxed set of specifications shown in Table 2. The system goal was to select a mirror figure error that would lead to a system wavefront error of about 0.07 waves rms (EUV), given a small allowance for residual design errors. The values for figure that were achieved differ from the specifications, but combine to approximately the desired value of wavefront error. MSFR and HSFR essentially achieve the interim goals, with MSFR for M2 being a little high $^{9}$. The Set 1 specification for HSFR is considerably above the Set 2 specification. The high level of HSFR reflects an area requiring additional process development in polishing. Of all the specifications for the substrates, however, HSFR has the least impact on performance, with an estimated incremental loss of $8 \%$ in reflectance per optic ${ }^{10}$. Since the MET is expected to have minimal demands on source power, this modest loss in throughput was felt to be acceptable.

Table 2. Figure and finish specifications for the MET optics and measured results for Set 1.

\begin{tabular}{|l|c|c|c|}
\hline & $\begin{array}{c}\text { Set 1 } \\
\text { Specs } \\
\text { [nm rms] }\end{array}$ & $\begin{array}{c}\text { Set 1 } \\
\text { Measured } \\
\text { [nm rms] }\end{array}$ & $\begin{array}{c}\text { Set 2 } \\
\text { Specs } \\
\text { [nm rms] }\end{array}$ \\
\hline $\begin{array}{l}\text { Figure } \\
\left(\mathrm{CA}^{-1}-\mathbf{1} \mathrm{mm}^{-1}\right)\end{array}$ & 0.33 & $\begin{array}{c}\mathrm{M} 1: 0.41 \\
\mathrm{M} 2: 0.25\end{array}$ & 0.25 \\
\hline $\begin{array}{l}\text { Mid-Spatial Frequency } \\
\text { Roughness (MSFR) } \\
\left(1 \mathrm{~mm}^{-1}-1 \boldsymbol{\mu m}^{-1}\right)\end{array}$ & 0.30 & $\begin{array}{c}\mathrm{M} 1: 0.23 \\
\mathrm{M} 2: 0.34\end{array}$ & 0.20 \\
\hline $\begin{array}{l}\text { High-Spatial Frequency } \\
\text { Roughness (HSFR) } \\
\left(1 \boldsymbol{\mu m}^{-1}-\mathbf{5 0} \boldsymbol{\mu m}^{-1}\right)\end{array}$ & 0.50 & $\begin{array}{c}\mathrm{M} 1: 0.49 \\
\mathrm{M} 2: 0.38\end{array}$ & 0.10 \\
\hline
\end{tabular}


Table 3. Key characteristics of the MET substrates.

\begin{tabular}{|lcc|}
\hline & $\begin{array}{c}\text { M1 } \\
\text { (primary) }\end{array}$ & $\begin{array}{c}\text { M2 } \\
\text { (secondary) }\end{array}$ \\
$\begin{array}{l}\text { Radius of } \\
\text { curvature }(\mathrm{mm})\end{array}$ & -318.13 & 340.67 \\
$\begin{array}{l}\text { Peak aspheric } \\
\text { departure }(\mu \mathrm{m})\end{array}$ & 3.82 & 5.61 \\
$\begin{array}{l}\text { Maximum aspheric } \\
\text { slope }(\mu \mathrm{m} / \mathrm{mm})\end{array}$ & -1.18 & -0.47 \\
$\begin{array}{l}\text { Clear aperture }(\mathrm{mm}) \\
\text { Angles of incidence }\end{array}$ & $\mathbf{8 . 4 - 2 7 . 0}$ & $11.4-91.6$ \\
& $2.54^{\circ}-8.67^{\circ}$ & $0.67^{\circ}-1.98^{\circ}$ \\
\hline
\end{tabular}

Figure 2 shows the interim status of the Set 2 optics as reported by Zeiss at the NGL Meeting in Pasadena, CA. " At this point, substrate M1 did not have a hole, while M2's hole was complete. Prior to machining the hole in M1, its figure value was driven below the specification value as a buffer in the event of distortion due to the drilling operation. M2 was nominally within the figure specification, although further polishing operations were planned to improve low order aberrations and perhaps the MSFR. As this report is being written, M2 is still undergoing improvement operations and M1 has had its hole drilled and is undergoing metrology.

One of the key metrics for evaluating the MET optics is the functional need for low flare. As mentioned earlier, both figure and MSFR contribute to flare. The frequency ranges associated with flare encompass those that lead to scattered light remaining within the printed field and are somewhat different for each of the optics as indicated in Table $4 .^{12}$ The integrated rms power in the extended-MSFR band for each optic using the interim data are presented in Table 4.

Table 4. Spatial-frequency ranges relevant to flare in the MET optical system and the integrated power in these bands using the interim data for Set 2.

\begin{tabular}{|c|c|c|c|}
\hline \multirow{2}{*}{$\begin{array}{c}\text { Extended Mid-Spatial } \\
\text { Frequency Range }\end{array}$} & $\begin{array}{c}\text { Integrated roughness over } \\
\text { extended range }\end{array}$ \\
\cline { 3 - 4 } & $\begin{array}{c}\text { Set 1 } \\
\text { (complete) }\end{array}$ & $\begin{array}{c}\text { Set 2 } \\
\text { (in-process) }\end{array}$ \\
\hline M1 & $0.11-160 \mathrm{~mm}^{-1}$ & $0.43 \mathrm{~nm} \mathrm{rms}$ & $0.20 \mathrm{~nm} \mathrm{rms}$ \\
\hline M2 & $0.031-50 \mathrm{~mm}^{-1}$ & $0.35 \mathrm{~nm} \mathrm{rms}$ & $0.16 \mathrm{~nm} \mathrm{rms}$ \\
\hline
\end{tabular}




\section{EUVL Micro Exposure Tool \\ Status of Zeiss Optics Fabrication}

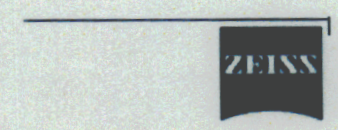

\begin{tabular}{l|cccc} 
& & & \multicolumn{2}{c}{ Actual Results } \\
& & & M1 & M2 \\
\hline Figure [nm rms] & 0.33 & 0.25 & 0.17 & 0.25 \\
MSFR [nm rms] & 0.35 & 0.20 & 0.24 & 0.22 \\
HSFR [nm rms] & 0.50 & 0.40 & 0.36 & 0.30
\end{tabular}
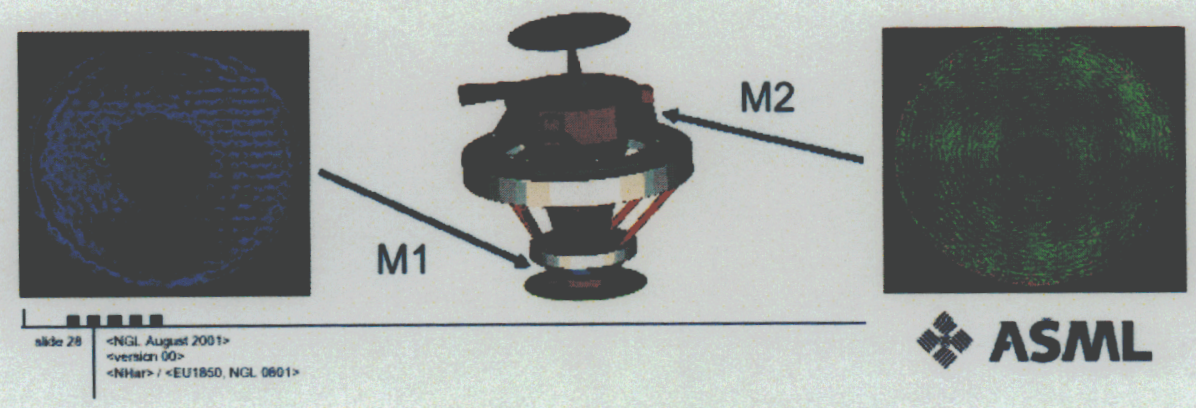

Figure 2. Status of the Set 2 substrates as reported by Carl Zeiss and ASML in September, 2001 at International Sematech's NGL Meeting in Pasadena, CA.

In Figure 3, flare is plotted versus linewidth for the Set 1 optics and the interim condition of the Set 2 optics. The flare is defined for an isolated dark line in a bright background with the field size as the dimensions of the MET printed field. Taking a cut-off linewidth of about $270 \mathrm{~nm}$, which corresponds to about 5 Airy diameters for $\mathrm{NA}=0.3$, the flare for Set 1 is about $22 \%$ and the flare for Set 2 is about $5 \%$. Thus Zeiss has been successful in improving the midfrequency waviness in moving from Set 1 to Set 2. Clearly, an important goal is to complete the Set 2 optics without increasing flare.

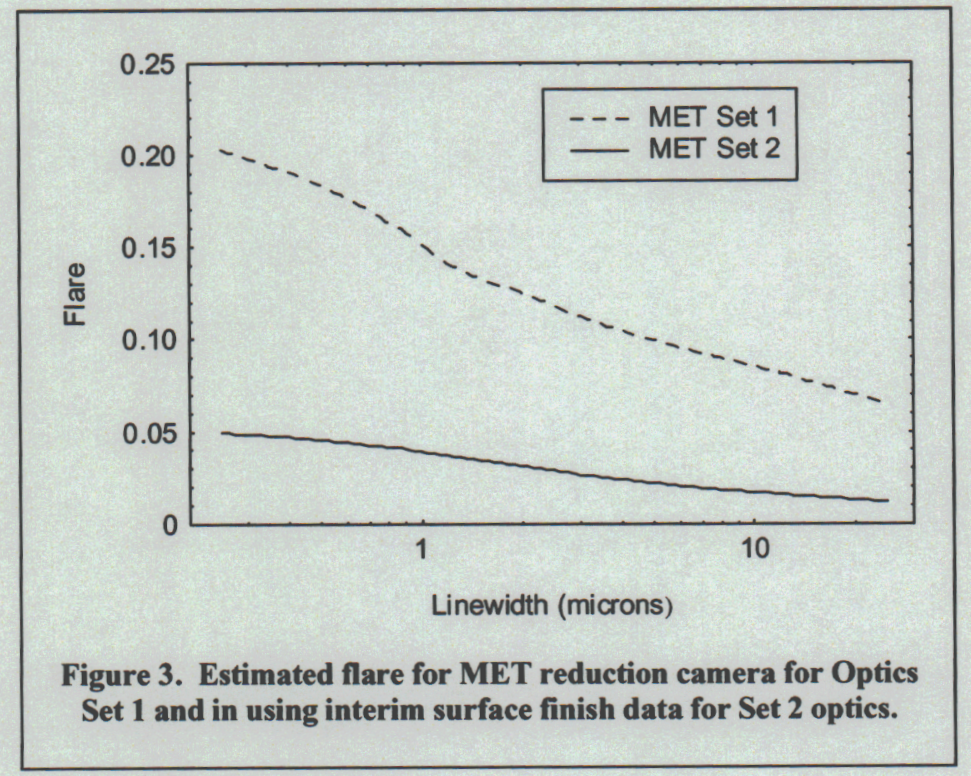

Page 7 


\section{Metrology Validation}

An important objective of Lith 112 is to validate the aspheric figure metrology that Zeiss will use in fabricating the MET substrates. To accomplish this, we have designed and constructed a Phase-Shifting Diffraction Interferometer (PSDI) at LLNL similar to those used in measuring the figure of the substrates for the Engineering Test Stand. Its accuracy has been extensively studied and significant experience has been gained in its use. Because of the PSDI's historical tie to accuracy and the fact that it is based on substantially different measurement principles, it is an ideal instrument for validating the accuracy of Zeiss's aspheric interferometer.

To accomplish the validation, both LLNL and Zeiss will measure an M1 substrate and then inter-compare the results. ${ }^{13}$ LLNL will use the PSDI and Zeiss will use its Direct $100^{\mathrm{TM}}$ interferometer that has been modified to accommodate the M1. Both instruments are designed to determine accuracy ${ }^{14}$ and are based on a first principles understanding of the intrinsic error sources. Each instrument is characterized by an error budget that provides an estimate of measurement uncertainty that asserts its ability to measure the MET optics. Our approach is to corroborate these error budgets by demonstrating that the difference between the Zeiss and LLNL measurements is small. The difference between the measurements must fall within the uncertainty derived from the error budgets. Assuming that the measurement uncertainty is sufficiently smaller than the figure error specification $(0.25 \mathrm{~nm} \mathrm{rms})$, then we will assert that the Zeiss interferometer can accurately certify the MET substrates to the required tolerances.

The overall plan for conducting this test was presented in December, 1999 as part of a design review on this effort. ${ }^{15}$ The $\mathrm{M} 1$ was chosen as the most appropriate element to use for the validation because it is considered more difficult to test than M2: it is convex and thus requires additional converger optics in the test cavity; and it has a higher aspheric slope than M2, leading to higher fringe density (see Table 3 ). The MET validation interferometer is installed within the same cavity as for the ETS M3 substrate, since both substrates are convex, face upward, and have similar radii of curvature. Modifications have been made to the M3 cavity to accommodate the aspheric profile of the MET optic (the ETS M3 is spherical) and the faster $\mathrm{f} / \#$ for the MET optic. One important difference between the measurement of the MET M1 and measurement of the ETS optics is that we mount the M1 into a rotation stage that can be rotated about the $\mathrm{M} 1$ axis between measurements. This provides the ability to use rotational averaging to eliminate essentially all interferometer errors that are not symmetric about the axis, and thus lower overall measurement error.

The basic optical layout of the interferometer is shown in Figure 4, which employs the same principles as the Phase-Shifting Diffraction Interferometers (PSDI) used to test the substrates for the Engineering Test Stand. ${ }^{16}$ Two nearly perfect spherical waves are generated by propaga ting laser light through a circular pinhole. The inherent accuracy of these spherical waves provides a first principles reference for comparison. The two waves, i.e., the test and the reference waves, are separated in time by inserting a delay leg in the reference beam (not shown). The test wave propagates to the converger, where it is brought to a virtual focus that lies at the center of curvature of the M1 mirror. This spherical wave reflects from $\mathrm{M} 1$ and acquires information regarding the aspheric shape and errors on the 


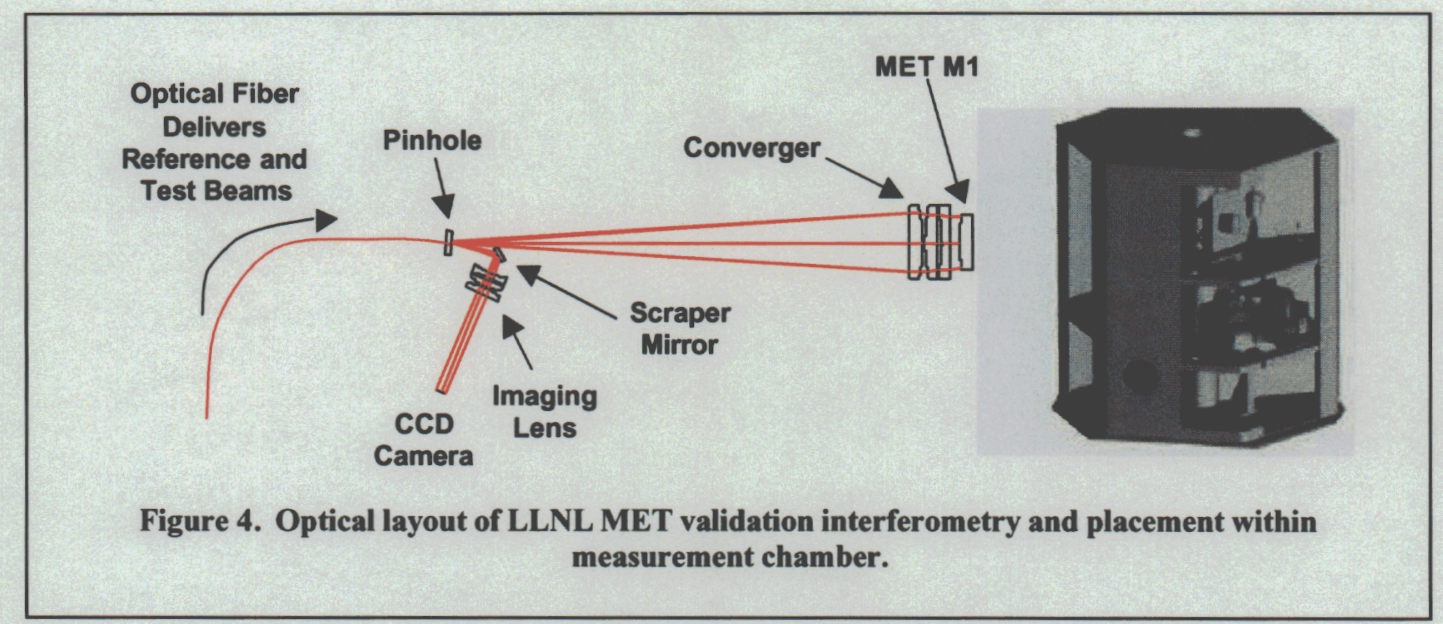

surface. The test wave propagates back through the converger and is focused onto the pinhole plane. At the time the test wave reaches the pinhole mirror, the reference wave is generated and joins the test beam, which is then intercepted by the scraper mirror. The two beams are directed into the imaging lens and then onto a CCD camera, where the interference pattern is recorded. The role of the imaging lens is to image the surface of M1 onto the CCD camera, so that there is a mapping between the coordinates of the test mirror and the locations of the CCD pixels. We were able to employ many of the procedures and analyses developed for measuring the ETS optics, such as the algorithms for correcting for the effects of dust within the system and the calibration of the converger lens.

We have performed a detailed error analysis of this interferometer for assessing its accuracy and will deliver a report to ISMT in Q4-01. The main contributions to errors include mapping and shear errors. Mapping errors arise due to small errors in linking the coordinates of the test surface to pixel locations on the CCD camera. Even when the height of the surface is correctly measured, if it is associated with the wrong $\mathrm{x}, \mathrm{y}$ location, there will be an apparent height error. Shear errors arise from the fact that the reference and test beams take slightly different paths to reach the CCD camera due mainly to the fact that the test beam contains the information about the aspheric contour of the surface and its errors. These error sources are discussed in detail in the upcoming report.

Two important components for calibrating the interferometer were fabricated and measured this year: a distortion calibration grid (DCG) and a measurement fiducial mask. The DCG, shown in Fig. 5, is a substrate with a radius of curvature similar to the test optic that has a precision set of fiducials (cross-hairs) deposited on the surface. A coordinate measuring machine (CMM) is used to measure the locations of the fiducials relative to each other and relative to the edges of the glass substrate. The DCG is then installed in the interferometer in place of the M1 mirror and the positions of the fiducials are observed relative to the camera pixels. Thus, a precise mapping is determined between the coordinates of the measurement plane and the camera pixels. This calibration process enables us to calibrate the small distortion errors in the interferometer's imaging optics due to fabrication errors, which is a primary contributor to the mapping errors mentioned above. ${ }^{17}$ 

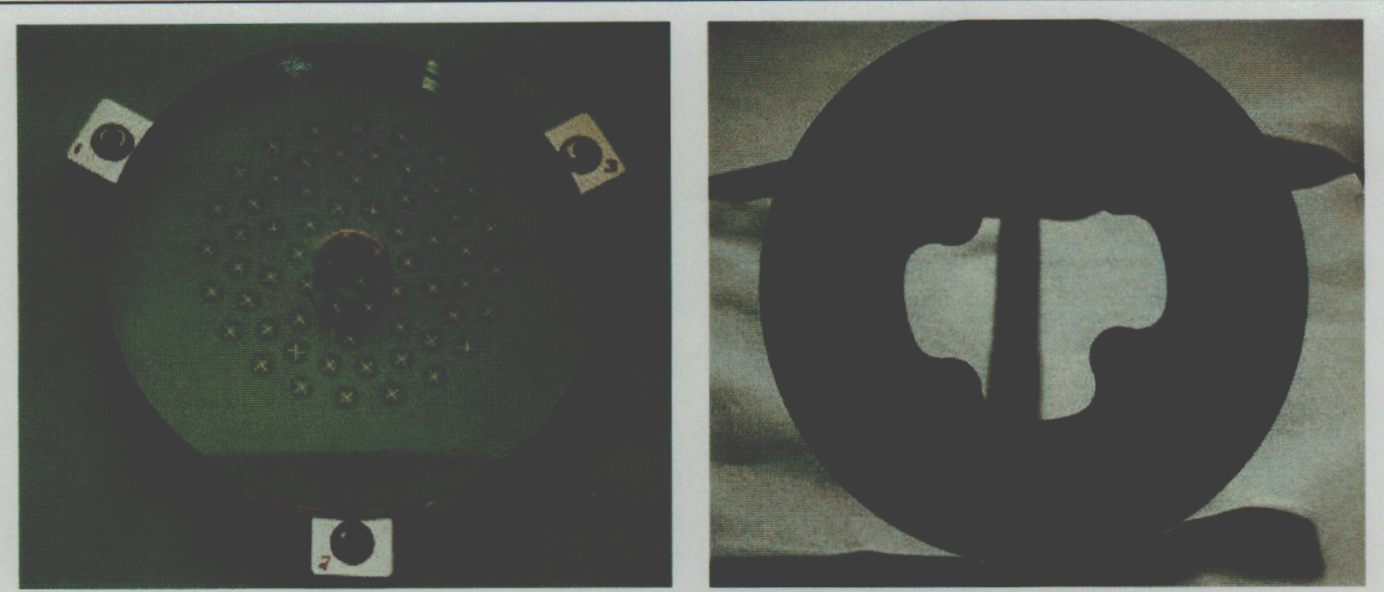

Figure 5. LEFT: Distortion calibration grid (DCG) used for mapping the coordinate system of the test optic to the CCD camera; RIGHT: Fiducial mask used for determining magnification and registering the interferometer to the substrate's datum surfaces.

The fiducial mask shown in Fig. 5 is a rigid thin metal mask that kinematically mounts on the test optic. The mask has precise cross-hairs machined into it whose spacing and location are carefully measured on a CMM. When an interferometric measurement is made of the test optic with the mask in place, the fiducials are visible within the measurement area and can be related to the geometric shape and size of the test optic. This enables us to associate the camera pixels with the fiducials, which are registered with respect to the edge of the glass substrate. ${ }^{18}$ This information is essential to assembling the optics with respect to one another by referencing the edges of the substrates. Also, imaging the distance between the fiducials with the CCD camera enables the system magnification to be easily determined. The fiducial mask is only in place during calibration and is removed when an actual figure measurement is performed.

During 2001, several M1 substrates have been measured during the process of calibrating and developing the error analysis of the LLNL interferometer. These parts have included relatively coarse substrates, the Set $1 \mathrm{M} 1$, and the final artifact used for the validation, M1-16. During March 2001, Zeiss metrology scientists visited LLNL to discuss the validation procedure and exchange information on aspheric metrology.

M1-16 is nominally fabricated to the Set 2 figure tolerances of $0.25 \mathrm{~nm}$ rms. This part is only intended for this validation of figure metrology and does not meet the finish specification. It was agreed between Zeiss and LLNL that M1-16 would be used prior to the completion of the Set 2 substrates in order to expedite the timely resolution of figure metrology issues while still having time to influence the fabrication process.

The final results of the validation measurements are presented in Figures 6 and 7. The height errors of M1-16 as measured by both LLNL and Zeiss are presented in Fig. 6. A somewhat broader spatial frequency range is included in the comparison than in the strict definition of figure. The same qualitative features are visible in each measurement. There is a distinct pattern of ripple and waviness, presumably from the signature of a small polishing 
tool. The outer edge shows azimuthal groove patterns. There is raised region near the center and several localized defects such as pits can be clearly distinguished. The fact that the Zeiss measurement of $0.22 \mathrm{~nm}$ rms is less than the LLNL measurement of $0.28 \mathrm{~nm} \mathrm{rms}$ is consistent with the fact that Zeiss was polishing the part to converge to zero error based on their own measurements, while the LLNL measurement shows the added influence of the systematic differences.

Additional information is obtained by considering the point-to-point difference between the measurements, as shown in Figure 7. This is an important measure for evaluating an interferometer because it considers both the magnitude and the location of the errors. As shown in the figure, much of the difference is rotationally symmetric, as might be expected since both measurement procedures make use of rotational averaging. The difference map has a magnitude of $0.25 \mathrm{~nm} \mathrm{rms}$. If the errors from the two interferometers are equal and statistically uncorrelated, and all systematic errors are represented by this difference, then each interferometer would be allocated an uncertainty of about $0.18 \mathrm{~nm} \mathrm{rms}$ (with a spatial frequency cut-off of 3.5 cycles per $\mathrm{mm}$ ).

If the difference between the measurements is filtered to the figure specification value of 1 cycle per $\mathrm{mm}$, then the difference drops to $0.22 \mathrm{~nm} \mathrm{rms}$. Applying the same assumptions as above, then each interferometer would be allocated an uncertainty in measuring figure of $0.16 \mathrm{~nm}$ rms. Given this assumption, then it may be concluded that the Zeiss interferometer is sufficiently accurate for certifying the MET substrates to $0.25 \mathrm{~nm}$ rms. Note that in applying this uncertainty measurement to the Zeiss interferometer, it would be suggested that the measured figure of M1 should be below $0.19 \mathrm{~nm} \mathrm{rms}$ to allow for the addition of measurement uncertainty and thus certify that the figure was less than $0.25 \mathrm{~nm} \mathrm{rms}{ }^{19}$

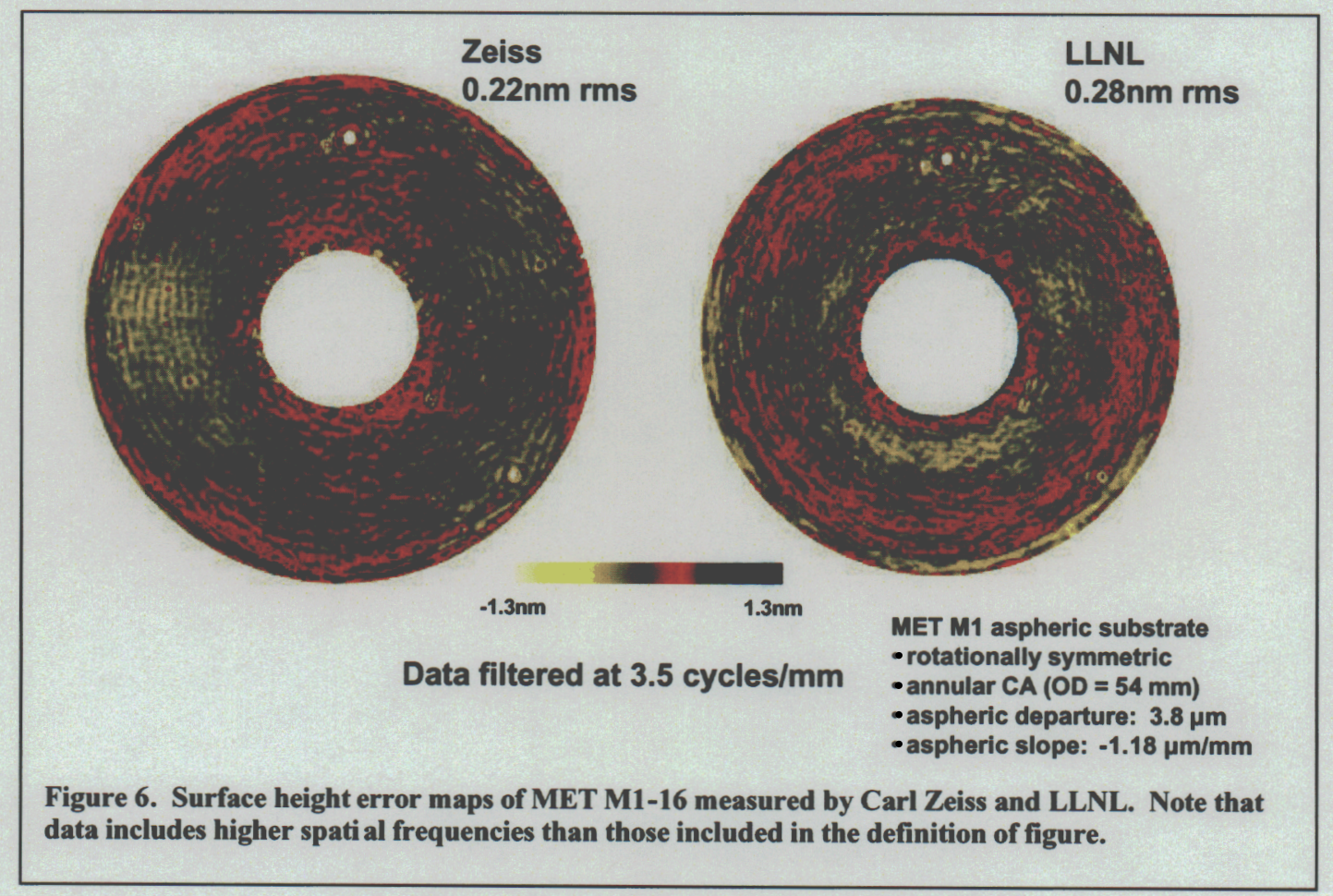


On the right side of Figure 7 is a plot of the average radial error of the difference map. There are well-defined structures indicating that a systematic azimuthal error exists in one or both of the interferometers. For example, any residual circular aberrations remaining from a converger calibration could be observed here. There is a deep ripple at the outer radius that is reminiscent of a circular fringe pattern that may have printed through to the final result. It is likely that errors with clear fingerprints such as these could be identified and removed with further analysis, but this is considered unnecessary in certifying the MET optics to the level of $0.25 \mathrm{~nm} \mathrm{rms}$.

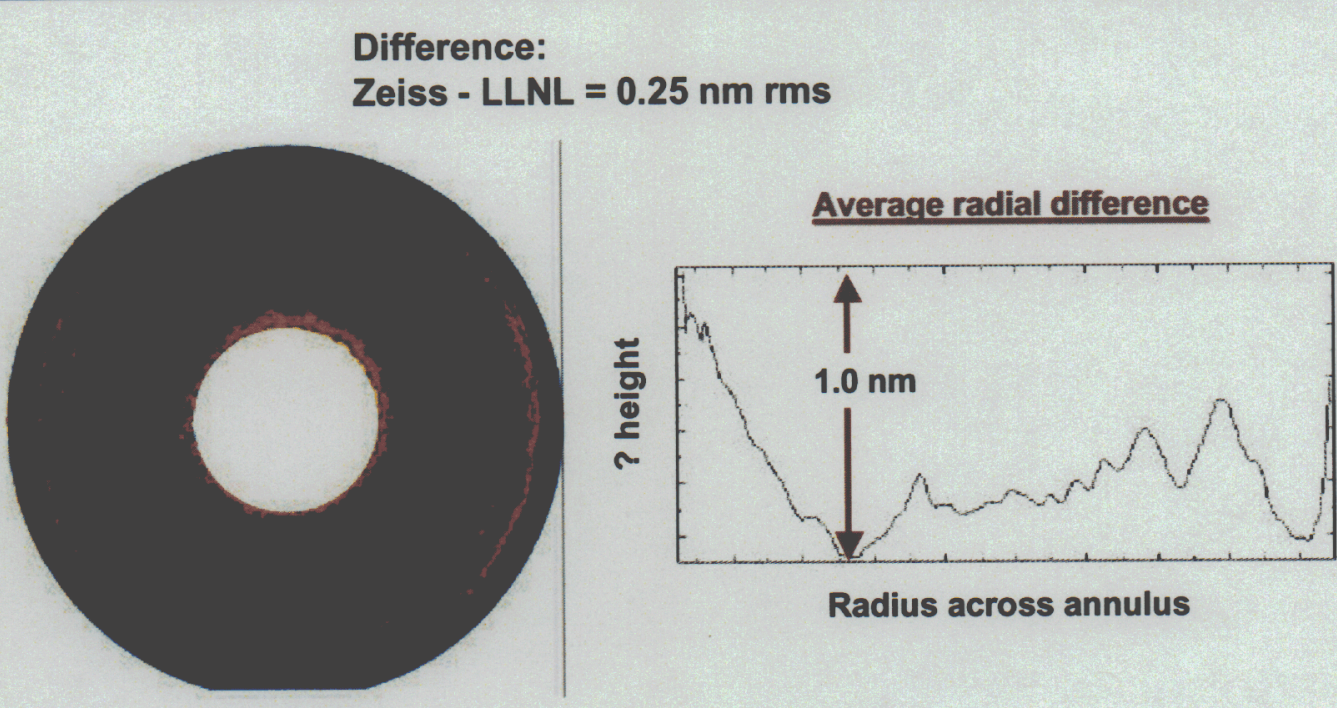

Data filtered at 3.5 cycles $/ \mathrm{mm}$

Figure 7. Map of point-to-point difference between LLNL and Zeiss measurements of MET M1-16.

Given that the Zeiss methodology for measuring M1 is validated, then the approach taken in this project is to consider the Zeiss approach for measuring M2 as similar and generally validated. In discussions with the Zeiss metrology scientists, there was a suitably detailed discussion of the error analyses for both M1 and M2 and it was clear that the same level of care was being applied to both elements.

Zeiss and LLNL have also collaborated on the cross-validation of metrology for surface finish. When Zeiss scientists visited LLNL in March 2001, many measurements were made on test optics using LLNL's phase shifting interferometric microscope (PMM) used for measuring MSFR and the AFM for measuring HSFR. Zeiss repeated these measurements on their own analogous instruments. Comparisons were made on the instrument readings, the analyzed data, error reduction strategies and analysis algorithms. In general there was good agreement, as indicated in the plots prepared by Zeiss in Figure 8 for PSDs determined from PMM measurements. Specifically, the agreement between the measurements is 
sufficient to validate the finish specifications for Lith 112. However, some differences in the data were not fully identified and are continuing to be studied. We hope to use scattering measurements at the ALS at LBNL to provide a functional calibration for these measurements. ${ }^{20}$

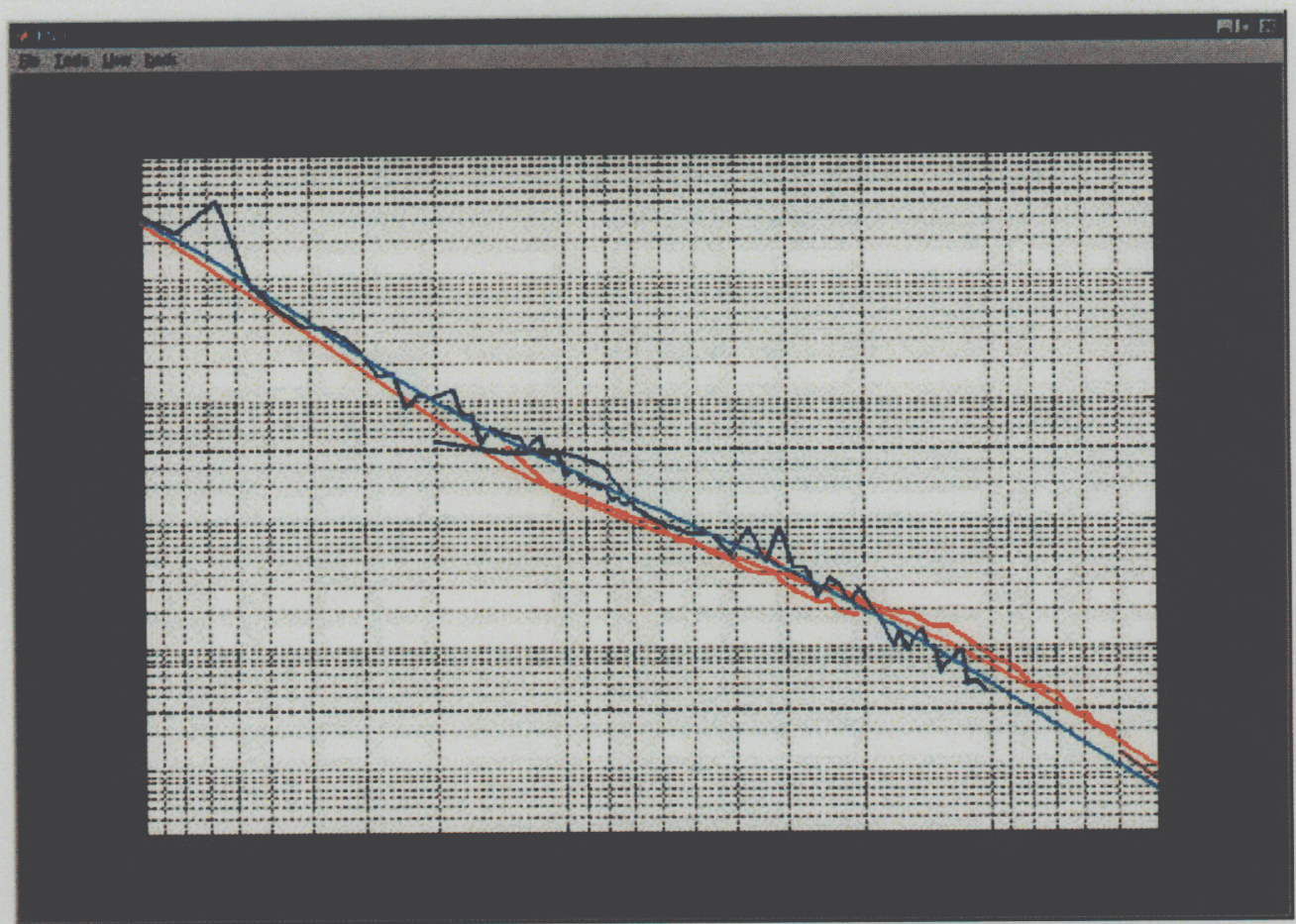

Figure 8. Comparison of surface finish PSD's measured by Zeiss and by LLNL; blue: Zeiss data, It. blue: fit to Zeiss data, red: LLNL data, orange: fit to LLNL data.

(plot from Frank Eisert, Carl Zeiss) 


\section{Multilayer Coating}

An essential element of the MET optics is the Mo/Si multilayer coating that enables the high reflectance of EUV light. These coatings comprise nominally 40 layer-pairs of molybdenum and silicon such that the combined thickness is one-half of the EUV wavelength (in that material). The functional specifications of these coatings are: ${ }^{21}$

1) maximize throughput by having a high reflectance and good wavelength matching among the optics

2) provide uniform reflectance across the optical surfaces so that coating-induced intensity variations across the camera exit pupil are negligible

3) induce negligible phase aberrations by minimizing the coating thickness errors across the optics

All of these functional requirements have been met on the MET Set 1 optics. We employed the newly acquired DC-magnetron sputtering tool "Mag4" shown in Fig. 9 that was also successfully used to coat the four ETS Set 2 optics. $^{22}$ A key feature of the Mag-4 deposition system is that it is designed to coat multiple optics during the same coating run in order to achieve essentially perfect wavelength matching among the optics. The rotating coating platter is also diagrammed in Fig. 9 and shows how the MET substrates are situated in two of the four stations used for the ETS optics. (There were no optics installed in the outer stations.) As the platter is rotating, each of the MET optics is simultaneously spun around their individual centers to average out spatial variations of the sputtering process and thus produce a coating that is symmetric (about the center of spinning). Also shown in the figure are the Mo and Si sputter targets over which the optics pass once for every layer pair.
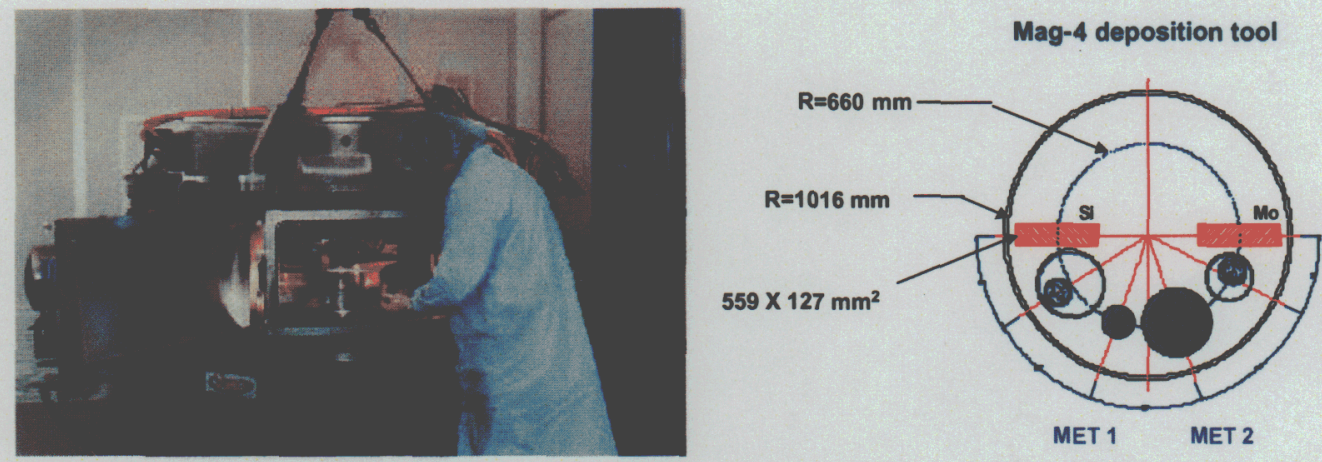

Figure 9. Both MET Set 1 optics were coated during the same run in the "Mag -4 " DC-magnetron sputtering tool.

A key to meeting the multilayer specifications is to employ our models of the coating process to determine the appropriate rotational speeds for the coating platter, as the optics pass under the targets. We also design and fabricate fixturing for holding the optics at precise locations and orientations with respect to the targets. We carried out the engineering to support these requirements in advance of receiving the Set 1 optics by 
adopting design principles similar to those used for the ETS optics. We then validated the fixturing designs and the prescription for rotating the optics using coating surrogates supplied by Carl Zeiss as part of their overall contract.

A requirement that is unique to fixturing the MET optics arises from the holes at the center of the optics. The M2 optic installed in its coating fixture is shown in Figure 10. The clear aperture (CA) of the optical surface extends directly to the edge of the hole, and it is important that any edge effects from the coating process, such as thickness gradients near the hole be minimized or they will affect image quality. This was accomplished by inserting a delrin plug into the hole such that its contour provided a nearly continuous surface at the edge of the hole. An added challenge for both designing and installing the plugs was provided by the existence of a small baffle located within the M2 hole to block the direct transmission of EUV light along the axis. The baffle in M2 and the delrin plug are shown in Figure 10.

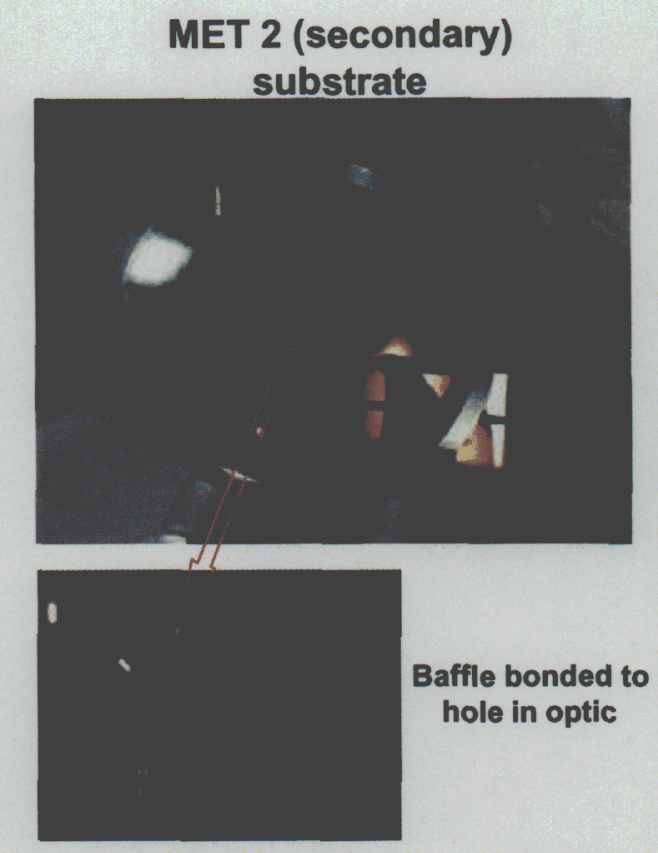

\section{Multilayer-coated MET 2}

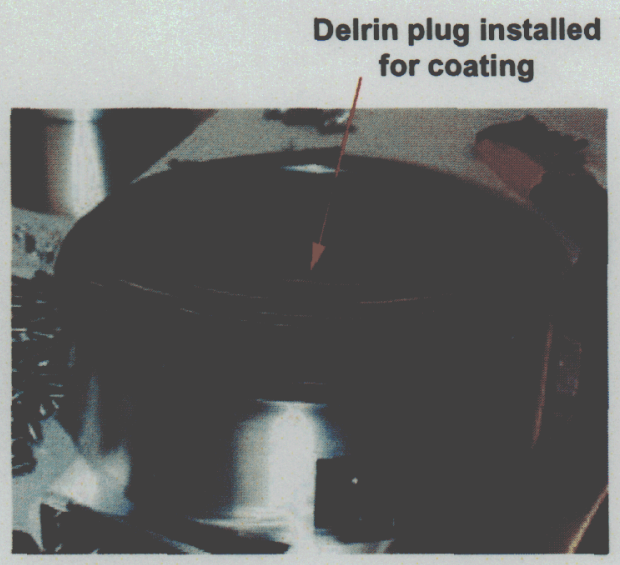

Figure 10. Ray clearance holes in the MET optics were filled during coating to minimize edge effects.

After the Set 1 coatings were complete, extensive metrology was performed at the ALS at LBNL. Figure 11 shows the M2 mounted in the reflectometer at the ALS. The M2 substrate (about $200 \mathrm{~mm}$ plus buttons) is the largest optic that can be accommodated in the reflectometer chamber. ${ }^{23}$ Reflectance versus wavelength is measured at multiple points on the optical surface at a fixed angle of incidence. The reflectance is then analytically adjusted for the actual angles of incidence at all locations on the surface. From this data, the peak reflectance, centroid wavelength, and uniformity can be determined. The remaining discussion of the MET multilayer coatings will summarize these results. 


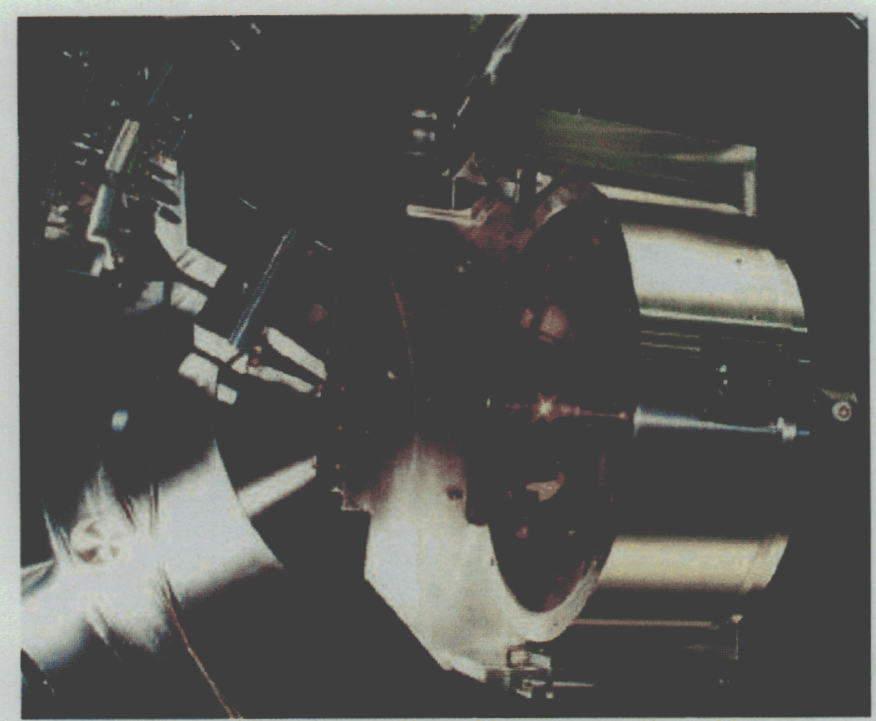

Figure 11. Special fixturing was used for measuring reflectance at the ALS at LBNL.

Figure 12 shows a measurement of reflectance versus wavelength for the Set 1 optics. $^{24}$ The two key parameters that are determined from these plots are the peak reflectance and the centroid wavelength. The centroid wavelength is defined as the wavelength at the center of the FWHM of the reflectance curve. Note that this data is collected at multiple locations on the surfaces so that variations in wavelength and reflectance across the surfaces can be characterized.
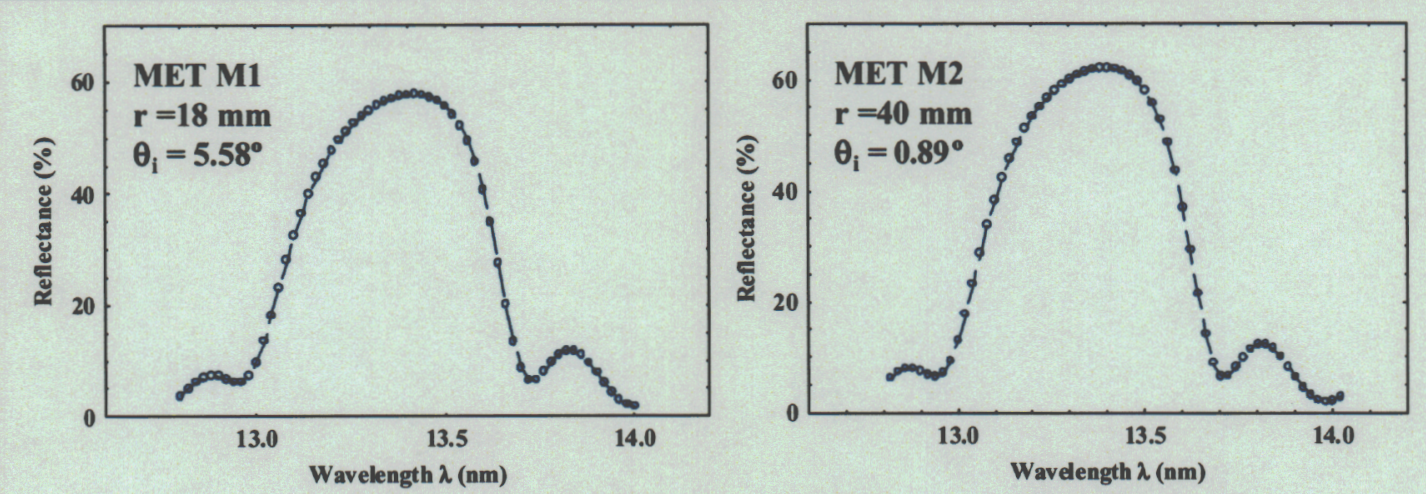

Figure 12. Measured reflectance curves on $M 1$ and $M 2$ for the angles of incidence within the camera at the specified radial locations. 
Figure 13 shows the centroid wavelength versus radius where the data are adjusted for the actual angles of incidence in the MET camera. This plot relates directly to the important functional specification of maintaining a uniform wavelength across the optical surface so that there is not a gradient in wavelength matching across the camera pupil. A specification level of $\pm 0.2 \%$ is shown on the plot, which is the same specification as for the ETS. For both optics, the measured values fall within the specification bars shown in Fig. 13. Also noted on the plot are the average values for the centroid wavelength that are essentially identical for the two optics. This provides a validation that coating both optics at the same time in the Mag-4 system can achieve excellent wavelength matching.
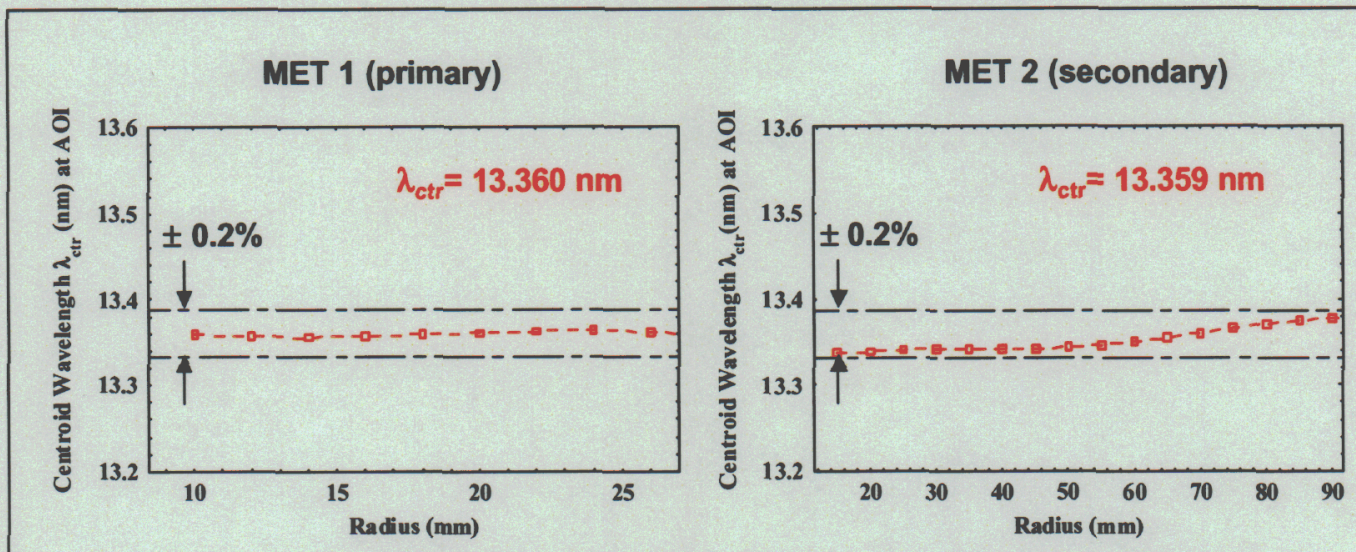

Figure 13. Excellent optic-to-optic w avelength matching was achieved for the Set 1 optics.

Variations in reflectance and centroid wavelength over the surfaces of the MET substrates are shown on the contour plots of Figure 14. The locations of the measurements were able to span the entire M1 substrate. However, for M2, the substrate's mounting buttons interfered with the stage in the reflectometer and precluded measurements within the three radial regions that are missing contour lines in the figure. It is evident in these wavelength maps that circular symmetry was achieved, which is indicative of our procedure for spinning the optics about their center.

The areal plot of reflectance in Fig. 14 shows a more irregular pattern where discrete regions have similar reflectance. We attribute this variation with variations in roughness (HSFR) on the substrate. These plots generally show a variation in reflectance of $<2 \%$ which suggests a variation in HSFR of $<1.0 \AA \mathrm{rms}$. This range of variation is consistent with the AFM measurements performed prior to coating. 

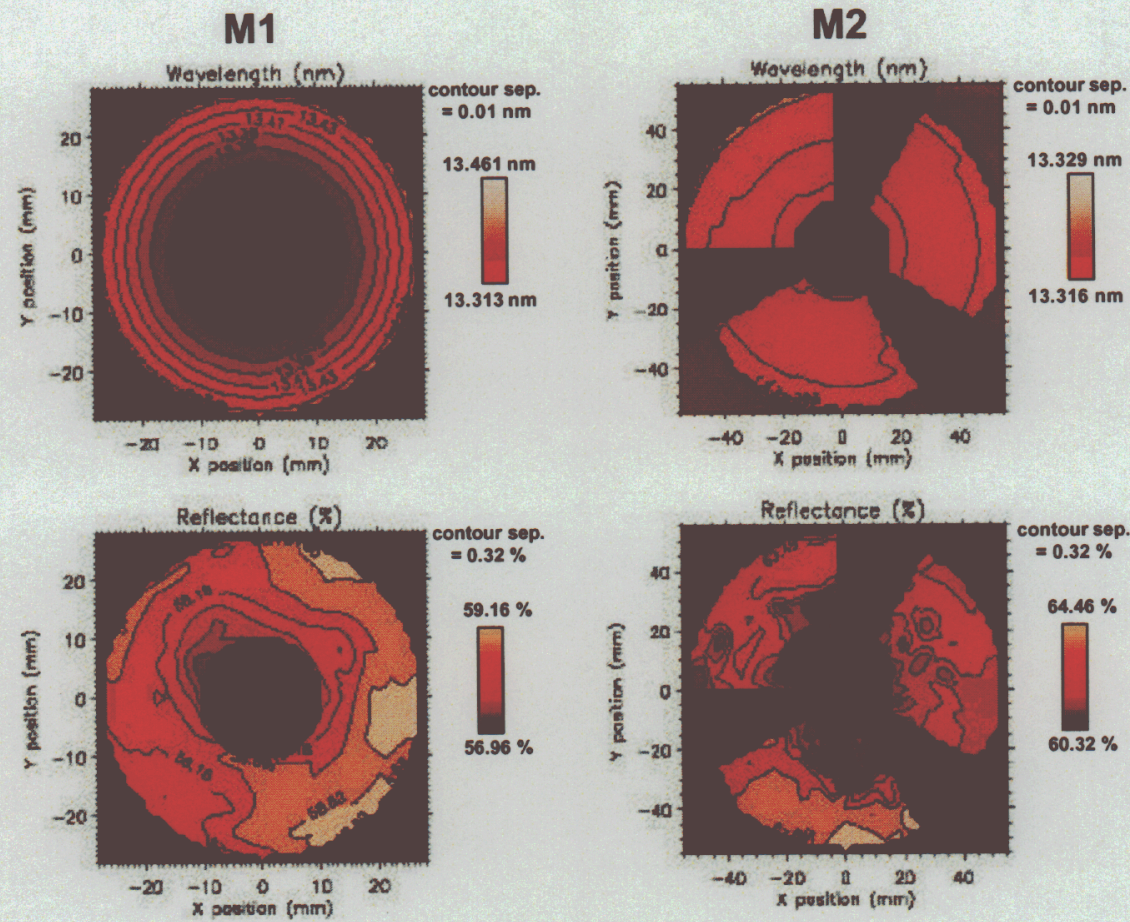

Figure 14. Areal maps of wavelength and reflectance were measured for the Set 1 optics.

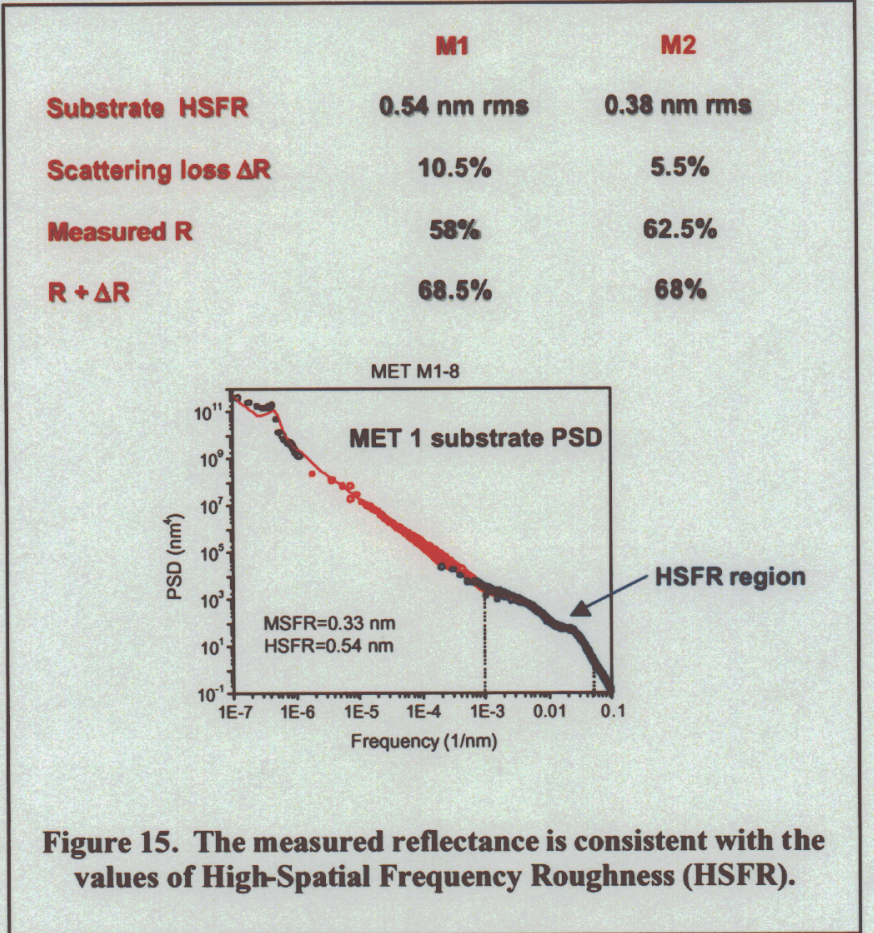

Page 18 
The average reflectance values of the Set 1 optics are given in Fig. 15 and are considerably below typical values of $65-68 \%$ for Zerodur ${ }^{\circledR}$ EUV optics. We associate this anticipated loss in reflectance with substrate roughness. We have been successful in predicting the reflectance of multilayers using a theoretical understanding of the multilayer growth process on rough substrates ${ }^{25}$ and the relationship between roughness and the angular distribution of scattering. ${ }^{26}$ In particular, we correlate the PSD of the surface roughness with scattered intensity. The PSD for M1 is shown in the figure, and the integrated roughness relevant to reflectance loss is about $0.54 \mathrm{~nm}$ rms. Our prediction for reflectance loss relative to a well-polished test surface is about $10.5 \%$. Thus, if we add our measured reflectance of $58 \%$ with the predicted loss of $10.5 \%$, we obtain $68.5 \%$. This would be the reflectance from an ideally smooth surface and has been verified experimentally from $\mathrm{Mo} / \mathrm{Si}$ coatings on superpolished flats ( $<1 \AA \mathrm{rms})$. As shown in the figure, there is an analogous link between reflectance loss and high roughness for M2.

As mentioned at the beginning of this section, a key requirement of the multilayer coatings is that they should not introduce significant phase errors into the reflected wavefront. In particular, it is desirable for the thickness of the multilayers to be sufficiently wellcontrolled that any added figure errors due to the coating be less than $0.1 \mathrm{~nm} \mathrm{rms}$. The

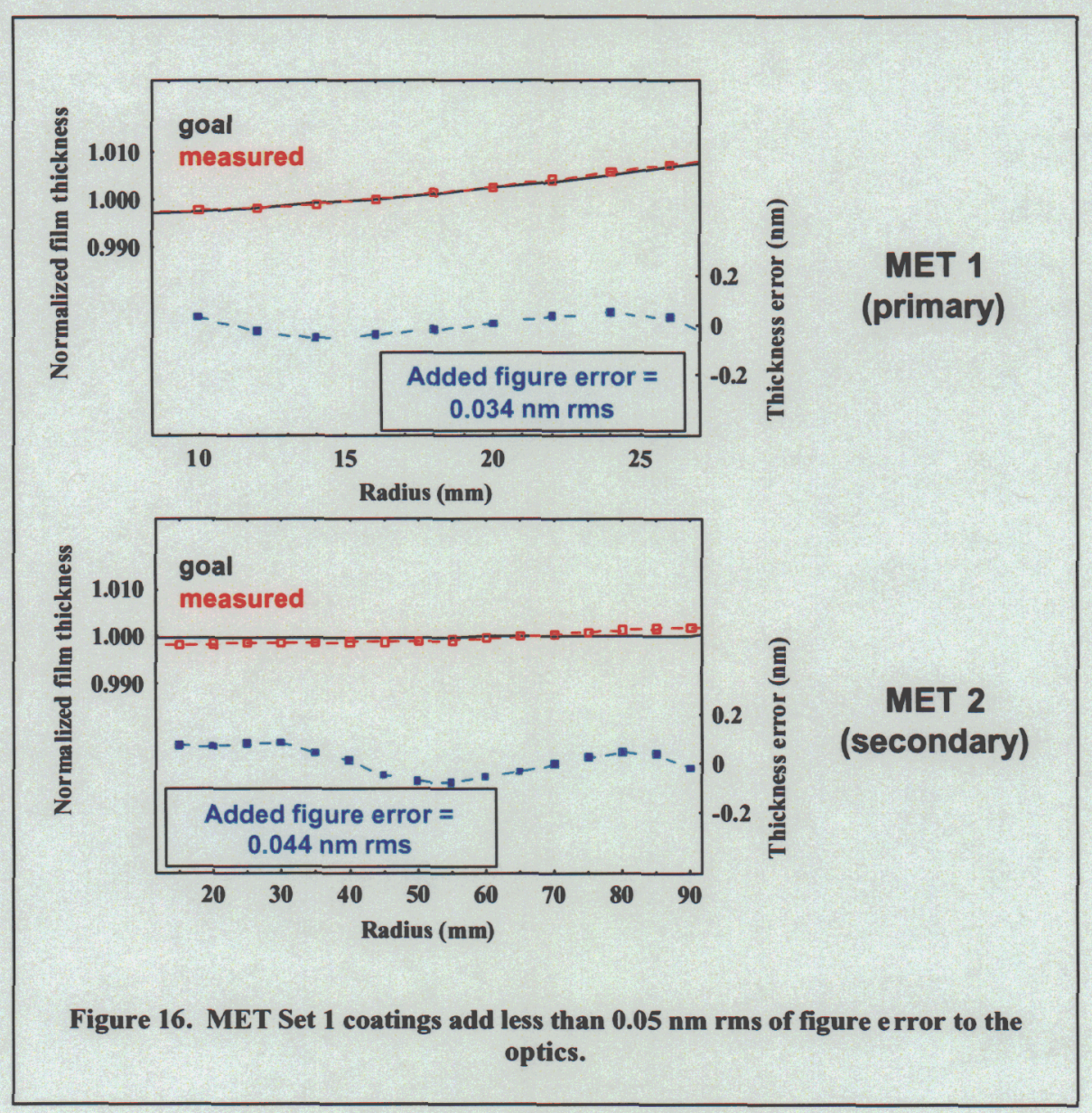


definition of the figure errors that are relevant here are those that are not readily compensable during PO Box alignment. Quadratic errors are easily compensable during alignment and are indistinguishable from simple radius errors. In Fig. 16, we plot for M1 and M2 the measured non-uniformity in comparison to the values predicted from the calculated prescriptions of the multilayer thickness profiles. The agreement is excellent. On the lower portion of the plots we have subtracted the compensable quadratic portion of the non-uniformity. These values for residual figure errors, $0.034 \mathrm{~nm} \mathrm{rms}$ and $0.044 \mathrm{~nm} \mathrm{rms}$, are very small in comparison with the goal of $0.1 \mathrm{~nm} \mathrm{rms}$ and the substrate figure errors listed in Table 2. We have a high level of confidence that similar low levels of coating-induced figure error will be attained for the Set 2 optics.

We have modeled the performance of PO Box 1 using ProLith ${ }^{\circledR}$ and the measured results for the multilayers. An example of CD vs. defocus is shown in Fig. 17 for dense $50 \mathrm{~nm}$ lines and spaces using the original MET optical design (no polishing induced aberrations are included since alignment data is not available). The simulations do not show any differences in $\mathrm{CD}$ behavior due to the presence of the multilayer coatings. Therefore, we conclude that the coatings do not degrade the performance of the system.

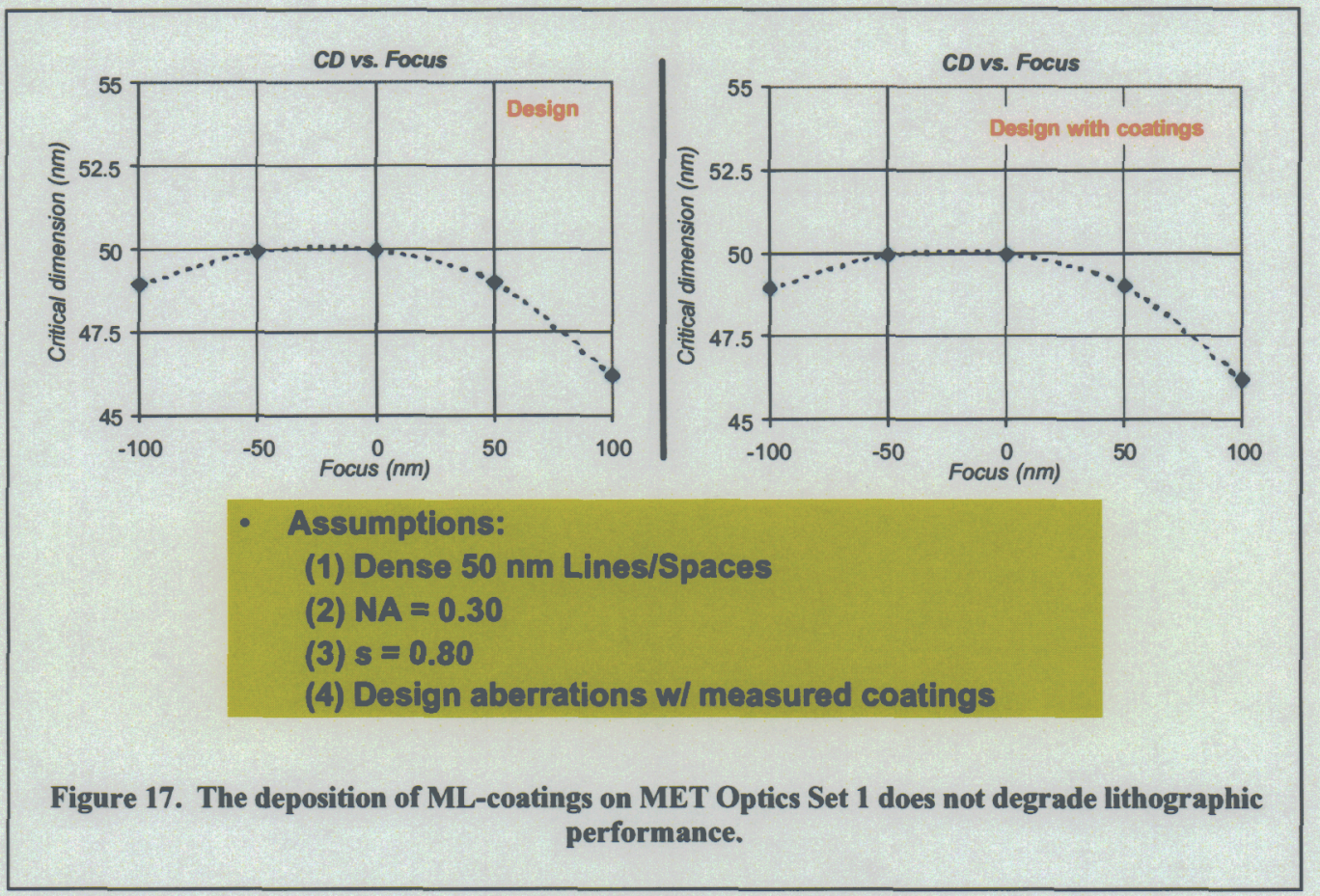




\section{Design, Assembly, and Delivery of PO Box 1 to Carl Zeiss}

During 2001, all components for PO Boxes 1 and 2 have been completed. With the delivery of the MET Set 1 optics, PO Box 1 was assembled, mechanically aligned, and shipped to Carl Zeiss in Oberkochen for interferometric alignment. Although PO Box 1 will stay with Carl Zeiss, it is timely and important to the completion of PO Box 2 because it demonstrates that the PO Box design meets its global requirements and validates that the shipping process is suitable for a PO Box with mounted optical elements. Also, as Zeiss proceeds with the interferometric alignment, there will be an important validation that all error budgets for optics manufacturing and assembly are consistent with the quality of the measured system wavefront error. PO Box 2 is ready for installation of the Set 2 optics when they are complete and coated with multilayers.

In this report, we will briefly review the key features of the PO Box ${ }^{27}$ design, including substrate mounting hardware, and describe the procedure used for assembling PO Box 1.

The MET PO Box was designed to meet several key objectives:

1) Provide a mounting for the optical substrates that exactly constrains all rigid-body degrees of freedom while minimizing disturbance forces that may distort the optic

2) Provide actuation of one optic relative to the other for alignment

3) Be robust to thermal and vibration disturbances

4) Provide datum surfaces for registering the optical axis with respect to mechanical features

5) Provide a kinematic mounting interface to other structures, such as an exposure tool or alignment interferometer.

All of these requirements have been incorporated into the design and are being demonstrated with PO Box 1. The details of the design of the PO Box have been presented during a formal design review in December 1999 with participation from ISMT and Carl Zeiss. ${ }^{28}$ Documentation of the design of the PO Box is provided in a milestone report to ISMT $^{29}$ and in conference papers. ${ }^{30,31}$

Figure 18 illustrates the key design features of the MET PO Box. All of the hardware is fabricated from SuperInvar, which has low thermal expansion for optimizing thermal stability. The PO Box Final Design Report describes the modal analysis performed in optimizing the dynamic response of the complete structure with the mounted optics. The lowest modes that result in significant relative motions of the optics are the M2 resonance frequency of $353 \mathrm{~Hz}$ and the M1 resonance frequency of $418 \mathrm{~Hz}$.

Each of the substrates is mounted to three buttons which attach to bipod flexures similar to those used for the ETS optics. The bipods have slots machined into them to form flexures that are rigid in tension or compression along their axes, but allow flexing in other directions. In this way, each bipod (which has two legs) constrains only two degrees of freedom. Thus each optic with three bipods exactly constrains six degrees of freedom: three for translation and three for rotation. This condition of exact constraint is essential 


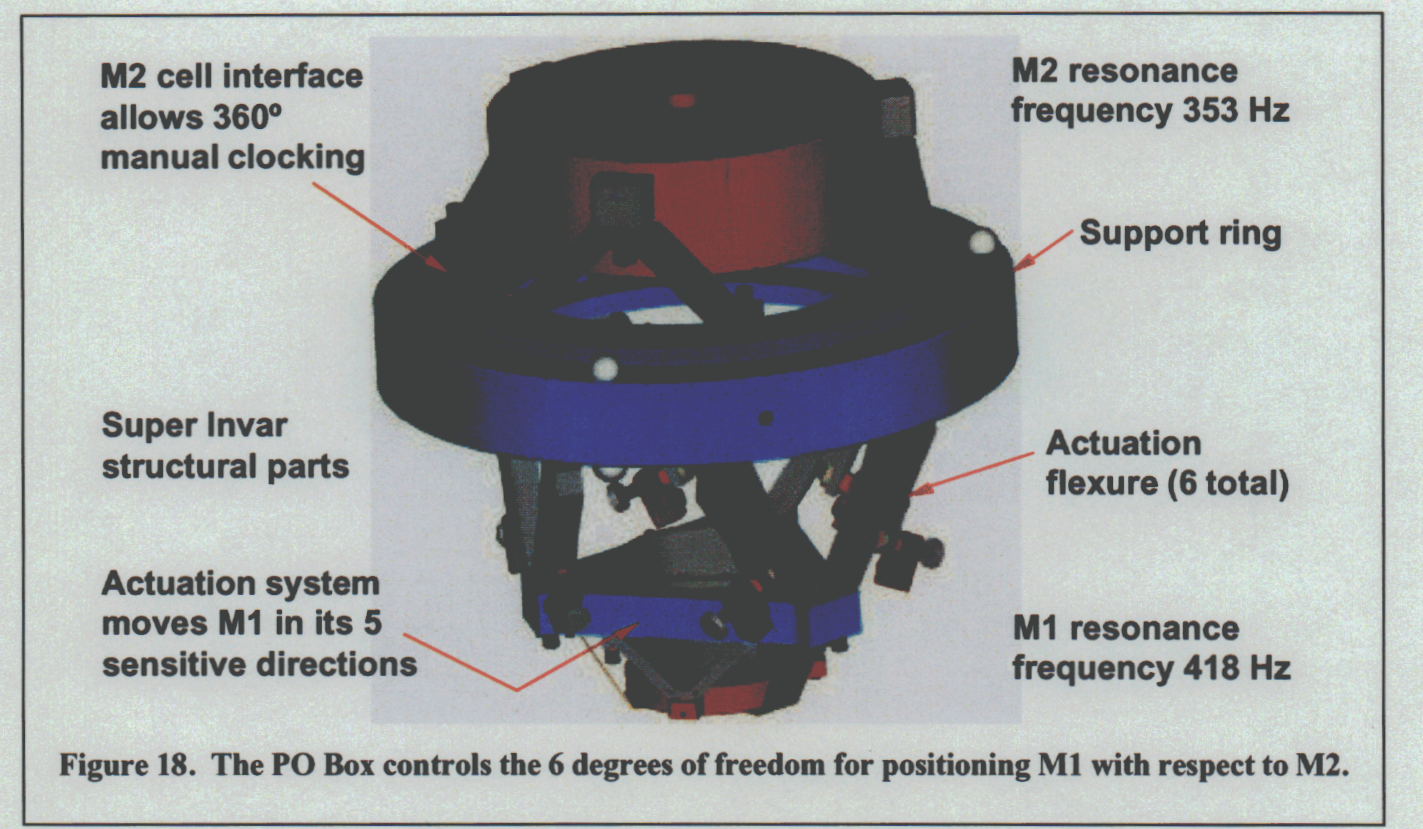

for minimizing distortion forces on the optics and in ensuring that the optics can be repeatedly inserted into their mounts and return to the same location.

The approach for aligning the optics is to hold M2 stationary with respect to the PO Box, while M1 is moved relative to the PO Box. The M1 bipod assembly hangs from the principal support ring by six actuation flexures. This is a well-known mechanical arrangement referred to as a Stewart platform. ${ }^{32} \mathrm{M} 1$ is positioned by adjusting the length of the actuation flexures (the mechanics of the flexures are described below). An extended degree of freedom is enabled by allowing the M2 bipod assembly to rotate within the support ring. This is provided to enable large clocking motions in aligning the substrates if the elemental interferometry suggests that aberration cancellation is offered by a particular relative orientation.

One of the early accomplishments in designing the PO Box mechanics was in developing an approach for using different mounts for interferometry and for installation into the PO Box. This approach was pursued for the MET because the optics needed to be held in the Zeiss interferometer, which did not have room for the bipods required by the PO Box. This is different than the procedure used for the ETS PO Box where the same mount was used for both the PO Box and interferometry, so that any mount-induced distortions would be identical for the two cases, and be automatically corrected during the polishing process. The technical challenge in designing the MET mounts is to ensure that any distortions induced by the mounts are either negligible or identical for the two types of mounts. ${ }^{33}$ The solution was in designing a mounting button for coupling the optics to the mounts that had a well-defined kinematic reference point that was common to the two types of mounts. As shown in Figure 19, the button that is epoxied to the side of the substrate has a spherical upper contour and a set of rounded corners on the bottom. These surfaces all reference a common spherical center. For the M1 shown in the left side of the figure, the rounded 
corners on the bottom of the buttons rest on cylindrical roller bearings for placement into the Zeiss interferometer. This relatively thin mounting structure meets the space constraint for the interferometer. For installation onto the PO Box bipods, the upper spherical surface is referenced. In each case, we strove to minimize the forces on the buttons. Roller bearing mounts were selected for the interferometer mount to minimize any residual frictional force that might arise in setting the optic into the mount. This approach was validated when Zeiss reported that they were able to remove and re-insert the optic into the mount with a reproducibility of $0.056 \mathrm{~nm}$ rms. Note that M2 employs a similar arrangement to that shown for M1.

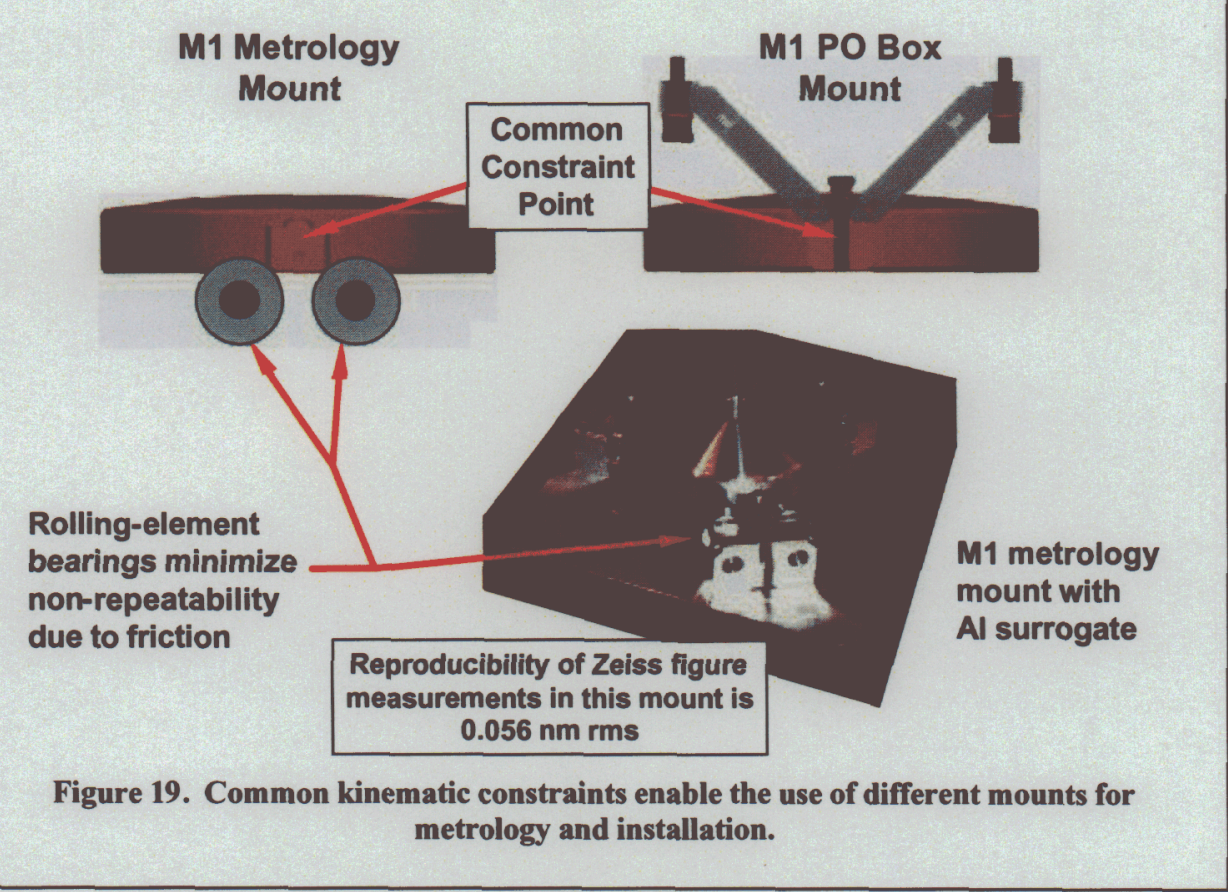

The development of the variable length struts that support M1 below the PO Box also was an important precision engineering accomplishment. It is required to provide stiff axial actuators for the Stewart-truss, yet also provide compliant flexures in non-axial directions. This was achieved with the design diagrammed in Figure 20. The axial direction of the flexures is indicated by the horizontal arrows labeled " 2 . Extension". To achieve an extension, i.e. lengthening, along the axis, the motion labeled "1. Actuation" shows a PicoMotor ${ }^{\mathrm{TM}}$ acting to separate two arms of a lever. As these two arms swing apart (only a minute distance), the hinge flexures (located at the right) rotate and cause the overall length to extend. A key advantage of the design is that there is a 10:1 reduction of the PicoMotor ${ }^{\mathrm{TM}}$ motion to axial motion. Thus, if the PicoMotor ${ }^{\mathrm{TM}}$ is commanded to move $100 \mathrm{~nm}$, then the axial length changes by only $10 \mathrm{~nm}$. This provides an important increase in the precision of the actuation. Also note that an LVDT is provided for actuator feedback, and that it can have less precision in the diagrammed orientation than if it was aligned along the axis. 


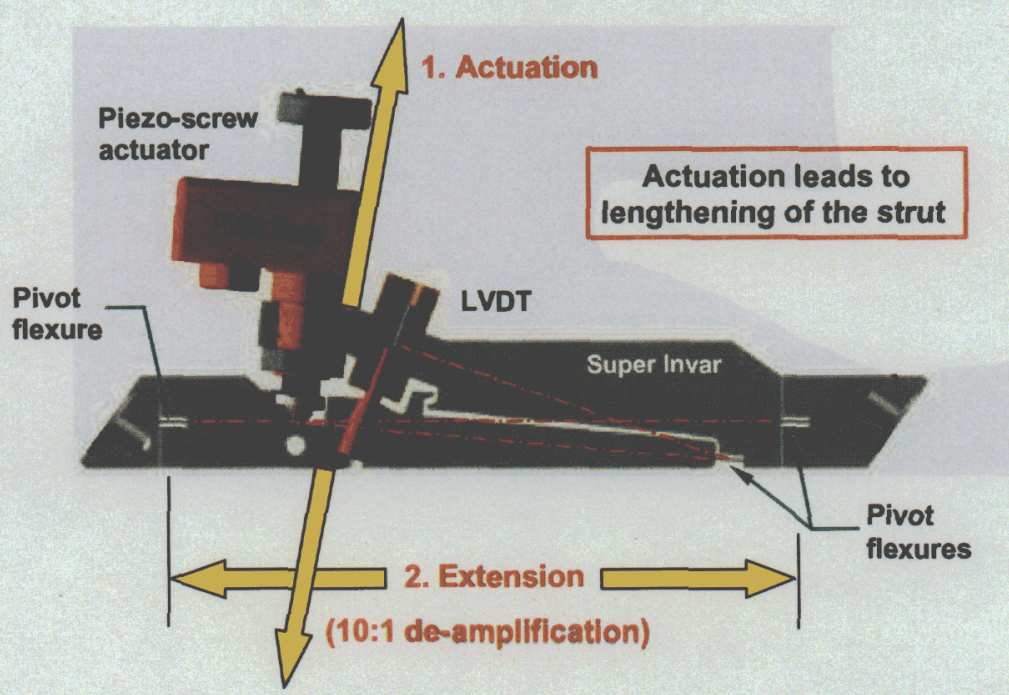

Figure 20. Strut arm length has sensitive control using pivot flexure design.

The procedure for assembling the MET PO Boxes comprises a number of delicate and detailed operations. ${ }^{34}$ The precision engineering has been carefully considered to preserve or establish coordinate relationships and to minimize the introduction of any nonrepeatable disturbance forces, such as fixturing friction. We have listed the key operations in Table 5, where many of the steps require measurements on a coordinate measuring machine. Figure 21 shows the CMM at LLNL where many of the assembly operations were performed.

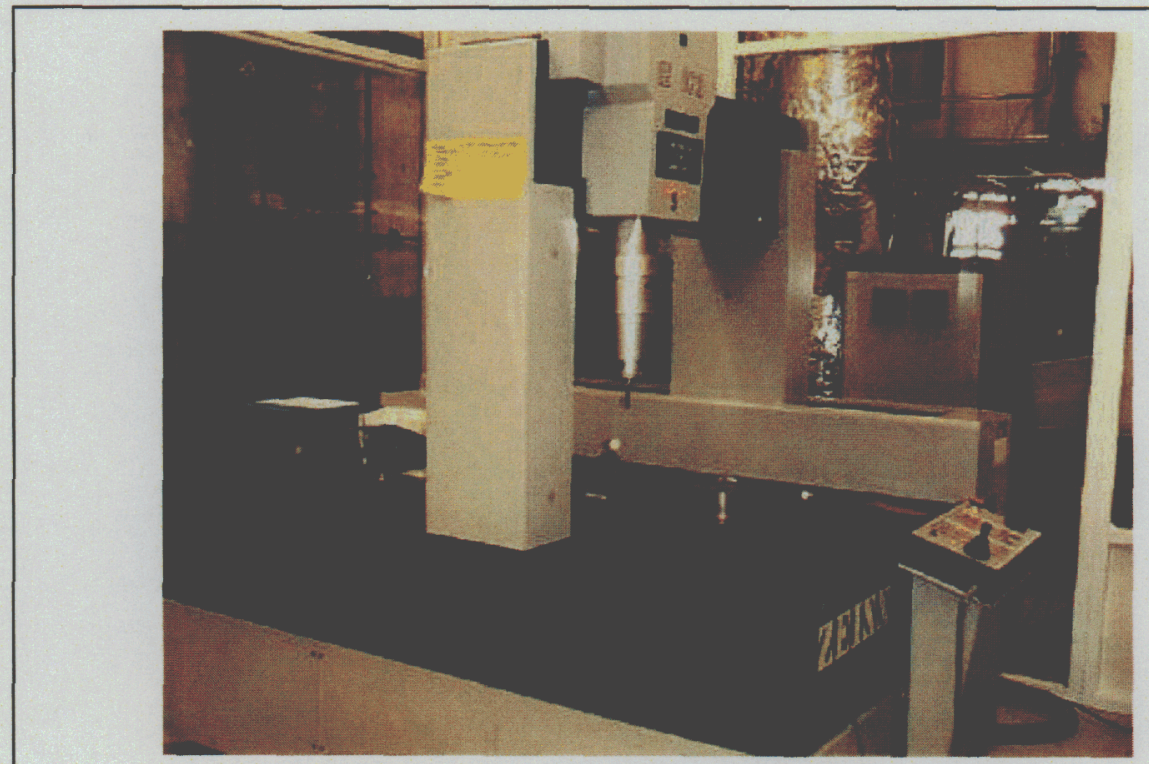

Figure 21. A coordinate measuring machine (CMM) is used for assembling the POBox. 
Table 5. Steps employed in assembling PO Box

\begin{tabular}{|l|l|}
\hline \multicolumn{2}{|l|}{ Use CMM to map the button locations to the aspheric coordinate system } \\
\hline & $\begin{array}{l}\text { Determine the vertex position on the substrate from interferometer } \\
\text { measurements provided by the optics vendor }\end{array}$ \\
\hline & $\begin{array}{l}\text { Probe the optical surface and the buttons with a CMM to determine } \\
\text { coordinate relationships }\end{array}$ \\
\hline & Transform the data to the best-fit aspheric coordinate system \\
\hline Set the optic mount to accept and properly place the optic \\
\hline & Assemble three flexures (bipods) to the optic cell set up on the CMM \\
\hline & Install a sphere into the conical set of each mount flexure \\
\hline & $\begin{array}{l}\text { Using the CMM, adjust the positions of flexure feet to move each sphere into } \\
\text { its prescribed position +/- 10 microns }\end{array}$ \\
\hline Set the location of the actuators to achieve mechanical alignment of optics \\
\hline & $\begin{array}{l}\text { Calibrate the LVDT through the range of motion of the actuation flexure and } \\
\text { set to mid travel }\end{array}$ \\
\hline $\begin{array}{l}\text { Attach the six actuation flexures to the support ring on one end and the M1 } \\
\text { cell on the other }\end{array}$ \\
\hline Machine spacers to achieve prescribed spacing and concentricity \\
\hline Install the optics into the mounts \\
\hline & $\begin{array}{l}\text { Using the CMM, measure four points on the top of each flexure to determine } \\
\text { its unstressed angle }\end{array}$ \\
\hline Lower the optic into the mount and secure (delicate procedure) \\
\hline Measure each flexure again to determine the rotation angles \\
\hline Compare to the error budget, repeat if necessary \\
\hline
\end{tabular}

The assembled PO Box is shown in Figures 22 and 23 where the M1 and M2 optics are obscured behind protective delrin covers, which are affixed to each optic in preparation for shipping. The main support ring is mounted on a display stand. The cables for the six actuators and position feedback sensors are clearly visible. Calibration of the system indicates that $10 \mathrm{~nm}$ positioning resolution has been achieved for alignment.

PO Box 1 has been shipped to Carl Zeiss in the shipping container designed by LLNL. ${ }^{35}$ Special attention was given to the g-forces that would be experienced in shipping and tests were performed to guarantee that the epoxy joints holding the buttons to the substrates would safely support the optics. The tests indicated the epoxy joints were robust and the subsequent shipment was successful. PO Box 1 is now at Zeiss awaiting completion of their alignment interferometer. 


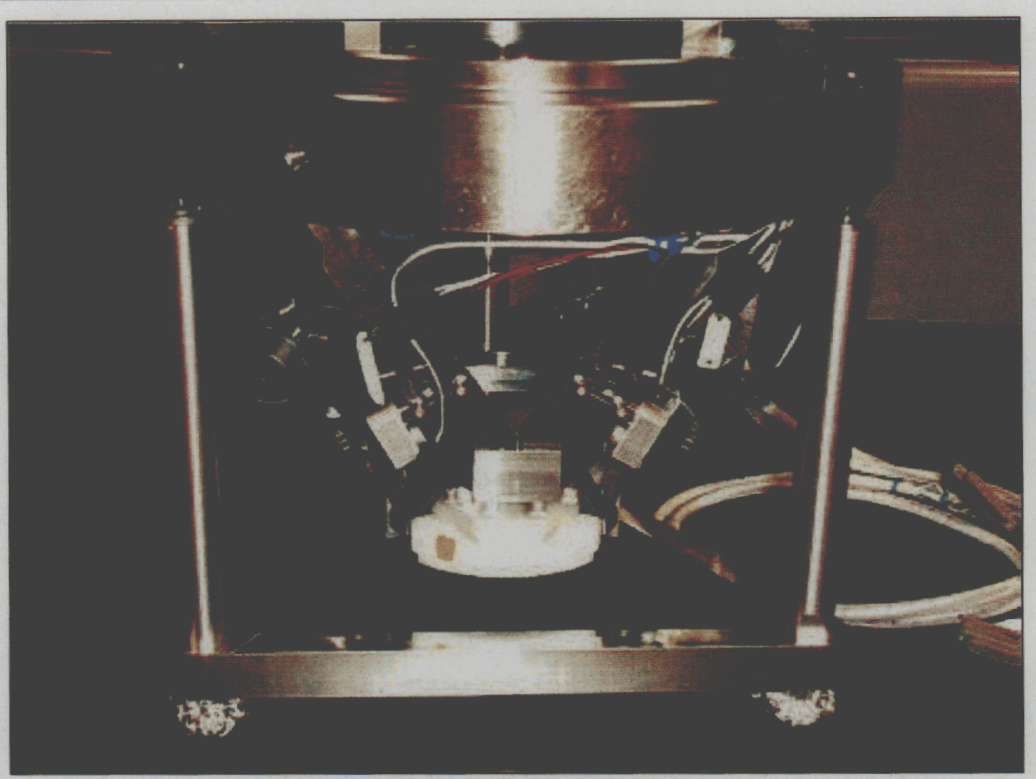

Figure 22. Remotely controlled actuators achieve $10 \mathrm{~nm}$ positioning resolution.

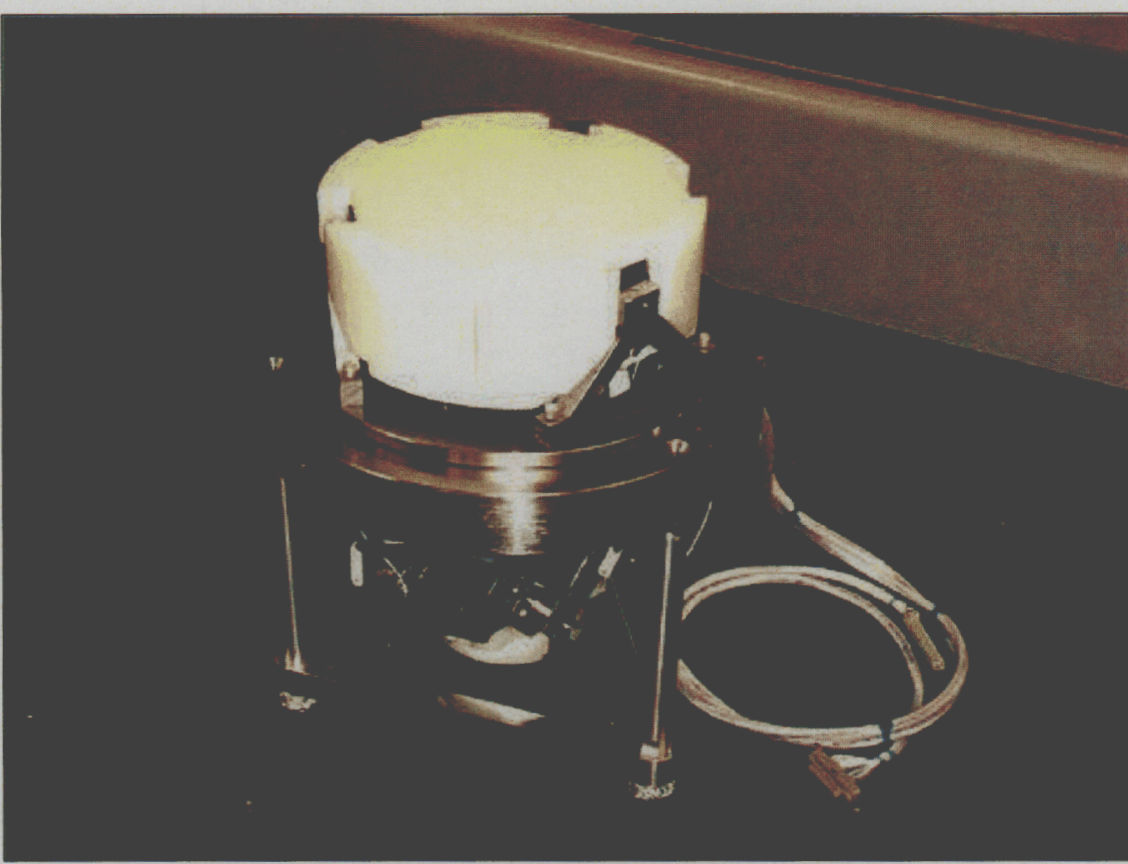

Figure 23. The Set 1 optics were installed into a PO Box at LLNL; photo shows the assembled PO Box with protective covers over the optics and accompanying sensoractuator control cables. 


\section{Summary}

Significant progress has been achieved toward constructing a 0.3 NA $5 x$ lithographic projection system for use in a small-field EUV Micro-Exposure Tool (MET). An optical design has been completed for the two aspheric mirror projection system that supports the printing of $30 \mathrm{~nm}$ dense features over a printed field of $200 \mu \mathrm{m} \times 600 \mu \mathrm{m}$. Carl Zeiss is under contract to fabricate the Zerodur $(\mathbb{B})$ substrates to excellent levels of figure and finish and then to align the assembled optical system. This contract also involves the assembly of interferometers for measuring the aspheric surface figure errors and a system interferometer for measuring system wavefront errors during alignment. A key aspect of the overall project is to validate Zeiss's figure metrology by comparison with measurements made at LLNL using the phase-shifting diffraction interferometer (PSDI). This validation has been completed using well-polished aspheric surrogates (M1) and the conclusions indicate that Zeiss interferometry meets accuracy requirements for validating the MET substrates.

An initial set of substrates, Set 1 , has been completed and is being used to demonstrate all steps in the construction and assembly process for an initial PO Box. The Set 1 optics have been coated at LLNL with $\mathrm{Mo} / \mathrm{Si}$ multilayers and have met stringent specifications for uniformity and wavelength matching. Reflectance is somewhat lower than typically attained on EUV optics due to residual high-spatial frequency roughness. The LLNL precision engineering group has designed and constructed a novel PO Box for supporting and actuating the optics. The Set 1 optics have been installed and mechanically-aligned into PO Box 1, which has been shipped to Carl Zeiss for interferometric alignment. Zeiss has been delayed in completing the Set 2 optics to the final specifications due to problems in developing a process to simultaneously meet both figure and finish specifications. We anticipate that the Set 2 substrates will be delivered to LLNL in Q4-01 for subsequent multilayer coating and assembly into PO Box 2 during Q1-02.

\section{Future Work}

The schedule for the completion of PO Box 2 depends on the completion of the substrates at Carl Zeiss. The key tasks remaining in completion of PO Box 2 include:

- Demonstrate alignment at Zeiss on PO Box 1 (Carl Zeiss)

- Complete fabrication and metrology of Set 2 optics (Carl Zeiss)

- ML-Coat Optics Set 2 (LLNL)

- Reflectometry of Optics Set 2 (LLNL-LBNL)

- Assembly of PO Box 2 (LLNL)

- Mechanical alignment of PO Box 2 (LLNL)

- Ship assembled PO Box 2 to Carl Zeiss

- Interferometric alignment of PO Box 2 (Carl Zeiss)

- Ship aligned PO Box 2 to LLNL/ISMT

- Calculate optical system performance using measured wavefront errors (LLNL-Paragon Optics) 


\section{Recommendations}

We recommend that if additional set(s) of MET substrates are acquired, the specifications for the optical surfaces be updated to reflect our increased understanding of their functional requirements. For example, we have determined which spatial frequencies contribute to flare and reflectance loss on each substrate, and Zeiss has gained knowledge on the feasibility of improving surface quality as function of spatial frequency. Adjusting the boundaries that separate figure, MSFR, and HSFR to reflect both functional needs and fabrication limitations may offer a path to improved overall performance.

The metrology validation process demonstrated that that Zeiss interferometry was capable of validating figure accuracy to the level of $0.25 \mathrm{~nm} \mathrm{rms}$. However, some distinct systematic differences remain between LLNL and Zeiss measurements that would likely be understood and reduced by further analysis. Continuing this metrology collaboration would ultimately lower the risk of fabricating either additional MET optics or lay groundwork for manufacturing optics for production tools. Similar collaboration in the area of finish measurements and their correlation with scattering measurements would lower the risk in meeting future specifications for flare and throughput.

\section{Acknowledgements}

The authors want to acknowledge the contributions from Sherry Baker for surface finish measurements, Lloyd Bradsher and Daren Dillon for interferometry, and Carl Chung for precision assembly. LLNL extends its appreciation to Frank Eisert of Carl Zeiss in helping analyze error sources in profilometry.

\section{Auspices}

This work was performed under the auspices of the U.S. Department of Energy by the University of California, Lawrence Livermore National Laboratory under Contract No. W7405-Eng-48. Funding was provided by the Extreme Ultraviolet Limited Liability Corporation under a Cooperative Research and Development Agreement. The authors also acknowledge support from International SEMATECH under project Lith 112. 


\section{Notes}

${ }^{1}$ Taylor, J. S., Sweeney, D., Hudyma, R., Hale, L., Kubiak, G., Sweatt, W., and Wester, N., "EUV MicroExposure Tool (MET) for near-term development using a high NA projection system", paper presented at the EUV Workshop held in Burlingame, CA, sponsored by International SEMATECH, October 19-21, 2000.

${ }^{2}$ Hudyma, R., "High NA microstepper final optical design report", Lith 112 report to Neil Wester at ISMT, Sept. 24, 1999.

${ }^{3}$ Hudyma, R., Taylor, J. S., Sweeney, D., Hale, L., Sweatt, W., Wester, N., "E-D characteristics and aberration sensitivity of the Micro-Exposure Tool (MET)", paper presented at the EUV Workshop held in Burlingame, CA, sponsored by International SEMATECH, October 19-21, 2000.

${ }^{4}$ Milestone Report for Nigh NA Optics Development, Lith 112, Milestone 4a: Specification package for the polished substrate M1, UCRL-ID-136162, Oct. 8, 1999.

${ }_{5}^{5}$ LLNL Drawing “Micro Exposure Tool, Projection Optics Box, Primary Mirror", AAA99-114289-0A.

${ }^{6}$ Milestone Report for Nigh NA Optics Development, Lith 112, Milestone 4b: Specification package for the polished substrate M2, Memo to Neil Wester, International Sematech, Dec. 7, 1999.

${ }^{7}$ LLNL Drawing "Micro Exposure Tool, Projection Optics Box, Mirror Button Assembly, Secondary", AAA99-114296-00.

${ }^{8}$ Taylor, J. S., Sommargren, G. E., Sweeney, D. W., and Hudyma, R. M., "The fabrication and testing of optics for EUV lithography", Emerging Lithographic Technologies II, Proc. of the SPIE Vol. 3331, pp. 580590 (1998).

${ }^{9}$ It is interesting to note what appears to be an inverse relationship between figure and MSFR: M1 has high figure and low MSFR, while M2 has the opposite. This may indicate the tradeoffs that are faced in simultaneously meeting both figure and finish.

${ }^{10}$ Mirkarimi, P. B., Bajt, S., Wall, M., "Mo/Si and Mo/Be multilayer thin films on Zerodur substrates for extreme ultraviolet lithography," Appl. Opt., vol. 39, 1617-1625 (2000); the sensitivity suggested in Fig. 3 of this reference is $-2 \% / \AA$. Thus a $4 \AA$ rms increase in roughness beyond the specification of $1 \AA$ rms would suggest an $8 \%$ incremental loss in reflectance. If the reflectance for a $1 \AA$ rms surface is nominally $68 \%$, then $60 \%$ should be expected for a value of HSFR of $5 \AA \mathrm{rms}$.

${ }^{11}$ Please contact ISMT for the availability of presentation material for the September 2001 Pasadena NGL Meeting.

12 There is an arbitrary distinction between the spatial periods that contribute to flare and those that contribute to figure aberrations. We take the low frequency boundary for flare to approximately correspond to scattering outside of five Airy disk radii.

${ }^{13}$ Note that LLNL is only measuring the M1, not the M2, since the goal here is to validate the metrology, not the optics.

${ }^{14}$ It is important to note that accuracy refers to the comparison of the optical surface to the analytical description of the ideal surface; most aspheric interferometers, however, measure only the precision with which a test surface matches a comparison artifact without assessing accuracy.

${ }^{15}$ Hudyma, R., "Metrology Validation Plan", Milestone report for SEMATECH Project Lith 112, delivered to Neil Wester, December 15, 1999, UCRL-ID-137249.

${ }^{16}$ Sommargren, G. E., Phillion, D. W., Campbell, E. W., "Sub-nanometer interferometry for aspheric mirror fabrication", from Precision Science and Technology for Perfect Surfaces, Proc. Of the $9^{\text {th }}$ International Conference on Precision Enginering (9th ICPE), Aug. 29-Sep. 1, 1999, Osaka, Japan, JSPE Publication Series No. 3.

${ }^{17}$ Distortion of the imaging lens is calculated analytically from the design of the lens. The DCG calibration process accounts for errors in fabricating the lens and constitutes a relatively small, but important correction. 18 There are carefully defined datum surfaces on the substrates for specifying coordinates on the surface.

$19 \sqrt{0.25^{2}-0.16^{2}}=0.192$

${ }^{20}$ Eric Gullikson (LBNL) has shown a good correlation between PSDs measured with profilometry and PSDs inferred from angle-resolved scattering measurements performed at the ALS at LBNL. Tests have been performed using EUV light at both normal incidence on multilayer-coated substrates and at grazingincidence on uncoated substrates.

${ }^{21}$ Hudyma, R., "Multilayer specifications for the MET projection camera", documentation of design milestone for Lith 112, Dec. 29, 1999; delivered to Neil Wester at ISMT. 
${ }^{22}$ Soufli, R., et. al., "Multilayer optics for an extreme ultraviolet lithography tool with $70 \mathrm{~nm}$ resolution," Proc. SPIE Vol. 4343, Emerging Lithographic Technologies V (2001); UCRL-JC-141186.

${ }^{23}$ Because the size of M2 with its mounting buttons is at the size limit of the reflectometer, there are some portions of the surface that are not directly accessible.

${ }^{24}$ This measurement is performed at a fixed angle of incidence to simplify the measurement procedure. The data is then adjusted to incorporate the actual angle of incidence for each radial location.

${ }^{25}$ Stearns, D. G., "Stochastic model for thin film growth and erosion", Appl. Phys. Lett., vol. 62, 1745-7 (1993).

${ }^{26}$ Gullikson, E. M., "Scattering from normal incidence EUV optics", Emerging Lithographic Technologies II, Proc. of the SPIE Vol. 3331, pp. 72-80 (1998).

${ }^{27}$ The jargonal term $P O B O x$ refers to the hardware that supports the optics, which in the current case resembles a truss structure instead of a box. $P O B O x$ is also commonly refers to the assembled PO Box, i.e. including the optics.

${ }^{28}$ Hale, L., "Conceptual Design Review: Micro Exposure Tool- Projection Optics Box", presentation package presented to Neil Wester of ISMT and Carl Zeiss, December 15, 1999; UCRLOVG-137252.

${ }^{29}$ Hale, L., "Final design report for the MET-POB", June 27, 2000, Lith 112 Milestone report sent to Neil Wester at ISMT.

${ }^{30}$ Hale, L., Hudyma, R., Taylor, J. S., Thigpen, R., Chung, C., and Chapman, H., "High NA camera for an EUVL microstepper", paper presented at the EUV Workshop held in Burlingame, CA, sponsored by International SEMATECH, October 19-21, 2000.

${ }^{31}$ Hale, L. C., Hudyma, R. M., Taylor, J. S., Thigpen, R. L., and Chung, C. A., "High-NA camera for an EUVL microstepper", Proc. of the Annual Meeting of the American Society for Precicion Engineering, Vol. 22 521-524 (2000).

${ }^{32}$ Stewart, D., “A Platform with Six Degrees of Freedom", UK Institution of Mechanical Engineers, Proceedings 1965-66, Vol 180, Pt 1, No 15.

${ }^{33}$ Hale, L., "Error budget for the MET optics supported in their metrology mounts", Lith 112 report to Neil Wester at ISMT, October 11, 1999; UCRL-MI-140015.

${ }^{34}$ For additional details on the assembly process or the underlying precision engineering, please contact Layton Hale (hale6@llnl.gov).

${ }^{35}$ Q4-00 Report for Lith 112 delivered to Pat Gabella at ISMT. 